\title{
Balkanologie
}

Balkanologie Revue d'études pluridisciplinaires

Vol. VIII, $n^{\circ} 1$ | 2004

Volume VIII Numéro 1

\section{Mitrovica/Mitrovicë : Géopolitique urbaine et présence internationale}

Mitrovica: The running of a conflict space by the international community

\section{Yann Braem}

\section{(2) OpenEdition}

12 Journals

Édition électronique

URL : http://journals.openedition.org/balkanologie/515

DOI : 10.4000/balkanologie. 515

ISSN : 1965-0582

Éditeur

Association française d'études sur les Balkans (Afebalk)

Édition imprimée

Date de publication : 1 juin 2004

ISSN : 1279-7952

\section{Référence électronique}

Yann Braem, « Mitrovica/Mitrovicë : Géopolitique urbaine et présence internationale », Balkanologie [En ligne], Vol. VIII, n 1 | 2004, mis en ligne le 21 janvier 2010, consulté le 17 décembre 2020. URL : http:// journals.openedition.org/balkanologie/515; DOI : https://doi.org/10.4000/balkanologie.515

(c) Tous droits réservés 


\title{
MITROVICA/MITROVICË GÉOPOLITIQUE URBAINE ET PRÉSENCE INTERNATIONALE
}

\author{
Yann Braem*
}

Parmi les espaces où les différentes communautés vivant au Kosovo sont en contact, Mitrovica/Mitrovicë ${ }^{1}$ occupe, à bien des égards, une place à part. Cette ville est en effet marquée par des phénomènes de ségrégation spatiale depuis plusieurs années ${ }^{2}$, qui persistent aujourd'hui, et comportent des répercussions politiques que notre contribution entend analyser 3 . La situation de ségrégation spatiale n'est pourtant pas inédite dans le Kosovo d'après-guerre. En

\footnotetext{
•Institut Français de Géopolitique - Université Paris VIII. (yabraem@wanadoo.fr)

${ }^{1}$ Nous utiliserons la double toponymie (serbe et albanaise) pour les noms de lieux que nous citerons dans l'article, et dans la mesure oủ ces données nous sont disponibles. Pour les cas dans lesquels nous n'avons pas la double toponymie, nous prions le lecteur de bien vouloir nous excuser, et d'accepter qu'un seul nom soit référencé, notamment pour les quartiers de la ville. Par ailleurs, nous utiliserons le nom Kosovo, dans le sens où il est utilisé dans les publications internationales, et non en suivant le nom serbe (Kosovo i Metohija) ou le féminin albanais (Kosovë).
}

${ }^{2}$ Roux (Michel), Les Albanais en Yougoslavie. Minorité Nationale, Territoire et Développement, Paris : Maison des Sciences de l'Homme, 1992.

3 Cet article s'appuie sur un ensemble de travaux de terrain effectués lors de séjour au Kosovo, à Mitrovica/Mitrovicë mais aussi à Priština/Prishtinë, en février 2001, avril et novembre 2002, qui permirent de mener des campagnes d'entretiens auprès des populations locales et des acteurs de la communauté internationale. Ces séjours se déroulèrent dans le cadre du DEA de Géopolitique effectué lors de l'année scolaire 2000/2001 au Centre de Recherches et d'Analyses Géopolitiques de l'Université Paris VIII, ainsi que dans le cadre d'une étude menée en 2002-2003, faisant l'objet d'une convention entre la Société de Géopolitique et le Centre d'Etudes en Sciences Sociales de la Défense (C2SD). Les résultats de ces travaux de terrain peuvent être retrouvés dans le mémoire de DEA soutenu en juin 2001 sous la Direction d'Yves Lacoste, intitulé Braem (Yann), Géopolitique d'une présence multinationale. La présence française au Kosovo face aux questions nationales, ainsi que dans le rapport du C2SD, Braem (Yann), Les relations Armées - ONG, des relations de pouvoir ? Caractéristiques et enjeux de la coopération civilo-militaire française: le cas du Kosovo, Paris : Documents du C2SD, 2004. Je tiens par ailleurs à chaleureusement remercier l'Organisation Non-Gouvernementale ACTED, ainsi que CARITAS-Secours Populaire, dont je fus hôte lors de mes séjours sur place, de même que le ministère de la Défense français pour sa coopération active. 
effet, aux lendemains de l'arrivée de l'OTAN sur cet espace, une partie des populations non-albanaises fuirent la Province 4 , en partie expulsées par des extrémistes albanais, en partie poussées par la peur d'actes de vengeance qu'ils auraient eu à subir en réponse aux exactions dont s'étaient rendues coupables les forces de sécurité serbes et yougoslaves durant la guerre de 1998-1999. La communauté internationale réagit à cette insécurité des minorités non-albanaises en accroissant le contrôle des zones de peuplement minoritaire par les troupes de l'OTAN, avant d'entamer un processus de retour des déplacés durant l'année $2003^{5}$.

Cependant, Mitrovica/Mitrovicë n'est pas dans une situation d'enclave, elle occupe la position de ville front entre des espaces de peuplement majoritaires serbe, les municipalités de Zvečan/Zveçan, de Zubin Potok et Leposavić/Leposaviq ; et ceux de peuplement majoritaire albanais, l'ensemble des autres municipalités du Kosovo ${ }^{6}$. C'est en partie pour cette raison, d'abord, que cette ville se trouve dans une situation de spécificité géopolitique, à l'instar de Brčko en Bosnie-Herzégovine? ${ }^{7}$. L'Ibar, la rivière qui traverse cet espace

4 En janvier 2003, l'UNHCR estimait à plus de 235000 le nombre de déplacés restant hors du Kosovo, majoritairement des Roms, des Serbes et Monténégrins.

5 Voir UNMIK, Manual for Sustainable Return, Priština, January 2003.

${ }^{6}$ La notion de peuplement majoritaire peut cependant être remise en cause. En effet, si l'on suit les résultats des élections locales dans chaque municipalitè, en prenant pour hypothèse que le vote s'effectue de manière largement communautaire, on peut observer que la municipalité de Štrpce/Shtërpcë a voté majoritairement en 2002 pour les partis serbes, ce qui suppose un peuplement serbe majoritaire, confirmant une tendance déjà remarquée dans le recensement de 1991. Le même phénomène est observable à Novo Brdo/Novobërdë où les votes exprimés en 2002 indiquent clairement que plus de $50 \%$ des voix ont été accordées aux partis serbes. Dans la municipalité de Gora/Dragash, cependant, les chiffres électoraux ne confirment pas la permanence d'une majorité de peuplement musulmane slavophone par un vote communautaire (voir les partis bosniaques dans l'OSCE, Political Party Guide, Priština, 2000), alors que le recensement de 1991 faisait apparaitre cette particularité. Nous reconnaissons cependant que l'hypothèse fondée sur les résultats électoraux s'appuie sur plusieurs a priori : une participation des populations aux processus électifs, une continuité entre l'appartenance communautaire et le vote en faveur de partis ouvertement communautaires. D'un autre point de vue, il est nécessaire de conserver à l'esprit que les mouvements de population ne sont pas arrêtés, tant du fait des menaces pesant sur la sécuritè des diverses minorités, que du fait du processus des retours engagé en 2002, ce qui implique de prendre une certaine distance avec des données observées sur un court terme. Enfin, la notion de peuplement majoritaire qui ne serait présente qu'au nord de l'Ibar est également une représentation géopolitique sur laquelle nous reviendrons, et qui fait l'objet d'une lutte de pouvoir entre les forces politiques serbes représentées dans les trois municipalités du nord et les forces politiques présentes dans les enclaves serbes, de sorte que le peuplement majoritaire indiquerait alors la continuité entre les aires de peuplement serbe du sud de la Serbie et du nord du Kosovo. Tout indique donc que l'on doit manier cette notion avec précaution, et nous l'utiliserons ici en prenant en considération un échelon de Province, sans aller jusqu'à l'échelon municipal, de sorte que l'on distingue deux grandes zones de peuplement serbes et albanaises.

7 Brčko se situe sur l'Inter-Entity Boundary Line, la ligne de séparation administrative entre les entités serbes et musulmanes instaurée suite aux Accords de Dayton en Bosnie-Herzégovine, et se structure de fait comme une ville front entre des territorialités antagonistes. Voir Ventura (Camille), " Le Corridor de Brčko (Bosnie), un litige territorial ", Géographie et Culture, (38), 2001 ; Sanguin (Jean-Louis), " La 
urbain, est ainsi un axe est-ouest de différenciation entre les diverses populations qui habitent le Kosovo, la division se retrouvant à l'intérieur même de la ville. Les Albanais occupent donc la partie sud de la ville et les Serbes, Roms et Bosniaques sont majoritaires au nord. Ensuite, c'est principalement dans cet espace urbain que les conflits sont les plus violents, opposant les populations serbes et albanaises entre elles, mais aussi ces mêmes populations aux diverses composantes de la communauté internationale ${ }^{8}$ (KFOR et MINUK principalement). Cette situation lui vaut une attention toute particulière de la part de la communauté internationale, attachée à la résolution des conflits, mais aussi de la part des autorités serbes et yougoslaves, qui envisageraient éventuellement que cette situation de ville-front se transforme en situation de ville frontière 9 , suivant un re-découpage territorial du Kosovo concomitant à une redéfinition de son statut politique.

$\mathrm{Si}$, globalement, la ville de Mitrovica/Mitrovicë est souvent présentée comme divisée en deux : une partie serbe et une partie albanaise, cette division sous-entendant que les zones de peuplements sont homogènes, on pourra observer qu'en fait, le sud de la ville seulement est homogène, à l'exception de six serbes vivant encore autours de l'Église orthodoxe. Le nord de la ville est, lui, fortement hétérogène, et soumis à des dynamiques de peuplement depuis l'arrivée de la KFOR et de la MINUK, dont les cartes disponibles ci-après permettent de rendre compte. On notera notamment deux éléments : la fin de toute présence serbe et rom au sud de la ville dès l'été 1999, et le retour d'un certain nombre d'Albanais dans leurs logements au nord de la ville selon des axes de circulation dont on resituera la logique. La population totale de la ville serait aujourd'hui d'environ 100000 habitants ${ }^{10}$. La répartition de cette population

Bosnie, Etat tricéphale des Balkans, les processus d'une partition ethno-politique ", Géographie et Culture, (38), 2001 ; Chaveneau-Lebrun (Emmanuelle), "La frontière inter-entité : nouvelle frontière, nouveau pays ", Balkanologie, 5 (1-2), 2001 ; Robin-Hunter (Laurence), "Brčko, un microcosme de la Bosnie ? ", Balkanologie, 5 (1-2), 2001.

${ }^{8}$ Des incidents réguliers sont rapportés depuis l'entrée des troupes de l'OTAN (KFOR : Kosovo FORce) à Mitrovica en juin 1999 et la mise en place de la Mission Intérimaire des Nations unies au Kosovo (voir l'encadré). Comme nous le verrons tout au long de cet article, les manifestations de violence peuvent mettre aux prises les populations albanaises et serbes entre elles, comme en septembre 1999 ou lors des récents événements de mars 2004, mais aussi les populations albanaises ou serbes contre la KFOR (février 2000, février 2001) ou contre la MINUK (avril 2002). Pour une chronologie des événements, voir, entre autres, le site du Courrier des Balkans : http://www.balkans.eu.org/ ainsi que celui de l'International War and Peace Reporting (IWPR) : http://www.iwpr.net/.

9 Voir les projets de recomposition de l'espace du Kosovo présentés par M. Roux (Roux (Michel), "Controverses sur les frontières du Kosovo ", Balkanologie, 7 (2), décembre 2003). Sur les notions de fronts et de frontières, voir Foucher (Michel), Fronts et Frontières, un tour du monde géopolitique, Paris : Fayard, 1991.

${ }^{10}$ International Crisis Group (ICG), “ UNMIK's Kosovo Albatros : tackling division in Mitrovica ”, Balkan report, (131), 3rd June 2002. 
serait la suivante : au nord, 12000 Serbes, 3000 Albanais, 2000 Bosniaques, 600 Turcs et 500 Roms; au sud, près de 80000 Albanais.

Cette première présentation du " cas géopolitique " particulier qu'est Mitrovica/Mitrovicë appelle une série de remarques. Tout d'abord, la géopolitique interne de la ville est soumise à des tensions liées au caractère central de la particularisation communautaire ou ethnique du peuplement, la plaçant au centre d'enjeux politiques, administratifs et sécuritaires. La violence s'exerce contre la " communauté ennemie ", en partie parce que sa présence n'est pas supportée, mais ne relève pas simplement du simple "voisinage " ou de l'aspect statique de la proximité du peuplement. Au contraire, la violence provoque des déplacements comme si elle en était le produit, le caractère dynamique du peuplement constituant un facteur aggravant des problèmes politiques de cet espace. Ensuite, cette violence ne s'exerce pas seulement contre la " communauté ennemie ", mais aussi contre les représentants de la communauté internationale. "Gérantes " de fait de cette Province du Kosovo, la MINUK et la KFOR, mais également l'OSCE et les ONG, sont interpellées, parfois très rudement, par leurs " administrés ", et ont de ce fait des difficultés à rendre compte de leurs problèmes dans la gestion du territoire. Ces difficultés, si elles ne semblent pas remettre en cause le bien fondé de la présence internationale au Kosovo, risquent toutefois d'engendrer un nombre croissant de conflits, posant la question des modalités d'intervention dans les zones de post-conflit ${ }^{11}$. Cette question se pose avec d'autant plus d'acuité que la légitimité des dirigeants politiques locaux, élus au suffrage universel, risque de rentrer en conflit avec la légitimité de la communauté internationale ${ }^{12}$, basée d'abord et avant tout sur l'action ${ }^{13}$.

\footnotetext{
${ }^{11}$ Il est d'ailleurs pertinent de se demander si l'on se situe bien ici dans une zone de post-conflit, dans la mesure où le mode de règlement des différents politiques entre les diverses communautés reste à bien des égards celui de la violence. Si toutefois le caractère de guerre n'est pas avéré, et parait quelque peu excessif, il n'en reste pas moins que l'on ne se situe pas dans une "société de paix n. La militarisation de l'espace urbain, de même que les incidents réguliers, ne peuvent que le confirmer, de sorte que l'on se demande si ce n'est le propre des situations de post-conflit caractérisées par une intervention de la communauté internationale, que de se situer dans cet entre-deux inconfortable. Voir les réflexions de $\mathrm{E}$. Luttwak, (Luttwak (Edward), " Give War a chance s, Foreign Affairs, July-August 1999) ; et M. Ignatieff (Ignatieff (Michael), " State Failure and Nation Building ", in Keohane (Robert O.), Holzgrefe (J. L.), eds., Humanitarian Intervention : Ethical, legal and political dilemmas, Cambridge : Cambridge University Press, 2003).
}

${ }^{12}$ Rupnik (Jacques), " L'avenir des protectorat internationaux dans les Balkans ", Critique Internationale, (16), juillet 2002. Voir également du même auteur "L'après-guerre dans les Balkans et la question du Kosovo ", Les Cahiers de Chaillot, (50), octobre 2001.

${ }^{13}$ Ainsí, la légitimité de la communauté internationale est d'abord liée au fait que son intervention a entraîné la " libération " du Kosovo, et donc à un certain registre de l'action. Voir Ghebali (Victor-Yves), " Totem et tabous dans le conflit du Kosovo ", Cultures et Conflits, (37), septembre 2000. 


\section{Chronologie non-exhaustive des principaux incidents survenus à Mitrovica/Mitrovicë depuis l'installation de la KFOR et de la MINUK [juin 1999]}

\section{JUIN/JUILLET 1999}

La MINUK et la KFOR prennent position à Mitrovica. L'hôpital est fortifié, mis sous administration conjointe MINUK/KFOR avant de passer sous contrôle civil à part entière. Le quartier rom est rasé maison par maison par des extrémistes albanais - les Serbes se sont enfuis du sud de la ville auparavant - pendant que la KFOR militarise le fleuve.

\section{SEPTEMBRE 1999}

Le 9 septembre, des manifestants albanais tentent de franchir le pont central qui enjambe l'Ibar. Ils sont retenus par la KFOR. Des manifestations violentes éclatent de part et d'autre. Les Serbes tentent d'expulser les Albanais de l'hôpital. Quelques mois après (décembre) la MINUK donne un ultimatum aux Serbes pour accepter la multi-ethnicité.

\section{FÉVRIER 2000}

Des attentats à la grenade sont perpétrés, contre un bus de l'UNHCR transportant des Serbes, puis dans un café de Mitrovica nord. Les Albanais vivant au nord de l'Ibar sont expulsés par les Serbes, les affrontements inter-ethniques et les manifestations s'accroissent puis des tirs de snipers visent la KFOR, visiblement de la part des Albanais. Le 20 février, l'Opération Ibar commence, avant de se terminer par un retrait de la KFOR.

\section{MARS 2000}

La Zone de Confiance est créée, mais l'hôpital de Mitrovica n'est plus géré par la MINUK, ce qui permet l'expulsion des Albanais et la prise de contrôle des médecins serbes.

\section{JANVIER/FÉVRIER 2001}

Suite à des incidents interethniques, et notamment la mort d'adolescents albanais, les populations albanaises manifestent devant le Centre Culturel et un bâtiment de la KFOR. Les manifestations dégénèrent très vite au sud, un immeuble de la KFOR est " caillassé ", les véhicules d'une ONG détruits et incendiés. Un attentat à Podujevo/Podujevë vise un bus de l'UNHCR faisant une dizaine de morts parmi les réfugiés serbes qu'il transportait.

\section{AVRIL 2002}

Le 8 avril, la MINUK police tente d'arrêter un des gardiens du pont, du côté serbe de l'Ibar, à une centaine de mètres du pont principal, au cours de ce qu'elle qualifiera 
de banale opération de contrôle de véhicule. La situation se dégrade fortement et rapidement; des coups de feu sont échangés de part et d'autres. La mobilisation extrêmement rapide et violente des miliciens, mais aussi de la population serbe, oblige les forces spéciales de la police à battre en retraite, alors que la KFOR gardant le pont ne bouge pas. Après cet incident, la MINUK et les organisations internationales ne se sont pas rendues au nord de la ville pendant plusieurs semaines. Durant cette période, des manifestations sont organisées quotidiennement par les Serbes dans les municipalités de Mitrovica/Mitrovicë, Zvečan/Zveçan, Zubin Potok et Leposavić/Leposaviq. Le renforcement de la mairie annexe de la MINUK au nord est reportée de plusieurs semaines.

\section{AOÛT 2003}

Des officiels de la Banque mondiale accompagnés du Premier ministre Rexhepi sont l'objet d'une attaque violente de la part de Serbes au nord de Mitrovica.

\section{MARS 2004}

Le 17 mars, un ensemble de manifestations sont organisées par les Serbes et les Albanais, notamment à Čaglavica (sud de Priština/Prishtinë). La situation dégénère rapidement dans l'ensemble du Kosovo et à Mitrovica/Mitrovicë, où les Albanais forcent les points de contrôle de la KFOR et pénètrent en masse au nord de la ville.

Faire une étude de la géopolitique interne et externe de la ville de Mitrovica/Mitrovicë semble donc être le préalable à l'examen de l'imbrication de la communauté internationale dans cette géopolitique. Le regard du " promeneur "14 que fut l'auteur de ces lignes en 2001 et 2002 doit ainsi être complété par un regard plus analytique, de sorte que l'on resitue l'observation dans une optique d'analyse géopolitique ${ }^{15}$. Tenter de comprendre une partie des stratégies politiques à l'œuvre permettra ainsi d'éclairer la position de la communauté internationale dans sa diversité, et d'illustrer à travers un cas pratique les difficultés de gestion des espaces de post-conflit ${ }^{16}$. Nous procéderons ainsi dans un premier temps en analysant l'espace urbain selon différents les

\footnotetext{
14 Aben (Jacques), « Une Géographie politique de Mitrovitsa ", Défense Nationale, (2), février 2003.

15 Pour une présentation des méthodes de la géopolitique, et notamment l'utilisation des différents niveaux d'analyse spatiale, voir Lacoste (Yves), La géographie, ça sert d'abord à faire la guerre, Paris : La Découverte, 1985 ; " Préambule ", in Lacoste (Yves), éd., Dictionnaire de Géopolitique, Paris : Flammarion, 1995 ; et " Hérodote a vingt-cinq ans, Ecologie et géopolitique en France n, Hérodote, (100), $1^{\text {er }}$ trimestre 2001.

${ }^{16}$ Rappelons brièvement ici l'intérēt que présente l'analyse des interactions entre populations locales et internationales dans les opérations de paix dont B. Pouligny fut, en France, précurseur (Pouligny (Béatrice), Les missions polyvalentes de maintien de la paix de l'ONU dans leur interaction avec les acteurs locaux : sociologie comparative de différentes situations : El Salvador, Cambodge, Haïti, Somalie,
} 
niveaux d'analyse spatiale que sont le quartier, la ville et la Province du Kosovo ainsi que son immédiat environnement régional, respectivement des ordres de grandeur du sixième ordre (quelques centaines de mètres d'envergure), du cinquième ordre (quelques kilomètres) et du quatrième (dizaines de kilomètres) et troisième ordre (centaine de kilomètres).

\section{GÉOPOLITIQUE DE MITROVICA/MITROVICË}

\section{L'échelon des quartiers}

Les quartiers au sud sont majoritairement des quartiers d'habitations individuelles ou d'immeubles peu élevés, à quatre ou cinq étages. Le trafic routier rythme la vie sonore de cette partie de la ville, avec ses klaxons, une densité très importante de véhicules individuels, transports en communs, laissant une impression un peu confuse de désordre faisant échos au débordement des échoppes sur les marchés ${ }^{17}$, regorgeant de denrées alimentaires, de matériel électronique, de biens de consommation courante ou ustensiles ménagers. Le quartier de la Yougo-Banka (voir carte) possède une urbanisation en décalage par rapport au reste de la ville, avec des immeubles de style plus " moderne " (une dizaine d'étages), dédiés aux centres commerciaux, centres administratifs. Il est traversé par quelques rues piétonnes bordées de restaurants. C'est dans ce quartier, le plus marqué par la présence militaire de l'OTAN au sud, que les composantes de la communauté internationale ont installé leurs bureaux ${ }^{18}$. Ce quartier est inclus dans la "zone de Confiance " instaurée au début de l'année 2000, une zone étroitement contrôlée par les forces militaires, destinée à être pluriethnique, et à permettre aux habitants serbes du nord de la ville de venir travailler au sud ${ }^{19}$.

Mozambique, Bosnie-Herzégovine, IEP Paris : Thèse de Doctorat, 1999, sous la direction de Bertrand Badie). Il nous semble particulièrement intéressant ici de croiser les analyses géopolitiques, dont l'intérêt pour les monographies régionales fait particulièrement sens dans les cas d'étude des processus de formation ethno-nationale, et les analyses plus orientées vers les relations internationales et qui visent à éclairer l'interventionnisme de la communauté internationale.

17 Ill existe deux marchés dans le centre sud de Mitrovica/Mitrovicë, celui situé en bordure est de l'ancien quartier rom, qui n'est pas permanent, et un autre, permanent celui-là, qui s'apparente à un " bazar ", situé au croisement des deux routes principales indiquées sur la carte au sud de la Yougo-Banka.

${ }^{18}$ L'UNHCR y a son bureau régional, comme la MINUK, l'OSCE, l'Union européenne ou encore le Kosovo Police Service.

19 Ce n'est pas sa seule fonction, voir plus bas. 
En se promenant au sud de la ville, on pourra observer qu'un certain nombre de zones ont été vidées de leurs populations. C'est le cas des quartiers situés à proximité de l'échangeur est de la ville, près du cimetière orthodoxe, qui étaient peuplés de Serbes et de Bosniaques. Leurs maisons furent occupées après la guerre par des familles albanaises. Le quartier Rom qui se situait plus à l'ouest en bordure de l'Ibar n'a pas eu la chance, lui, de " survivre " à l'aprèsguerre. Dès l'été 1999, les maisons furent vidées de leurs habitants et rasées, une par une, par des extrémistes albanais ${ }^{20}$. L'église orthodoxe reste habitée par quelques Serbes, notamment le prêtre, et est fortifiée, protégée jours et nuits par la KFOR. Ces lieux de culte, l'église et le cimetière, sont des lieux à forte dimension symbolique pour les populations serbes, qui demandent à pouvoir s'y rendre lors des fêtes religieuses.

Au nord de la ville, la géographie du peuplement est nettement plus hétérogène. La Petite Bosnie, ou Bosna Mahala (ou Mala Bosna), par exemple, est le quartier abritant de manière traditionnelle les populations albanaises et musulmanes slaves. C'est un quartier pauvre, délabré, avec de petites maisons, où l'armée française est présente en permanence, patrouillant dans les rues pour préserver la sécurité d'une zone qui connaît régulièrement des flambées de violence. Ce quartier fait aussi partie de la zone de Confiance, qui est ici destinée à sécuriser l'accès avec le sud de la ville (deux ponts se situent à proximité de la Petite Bosnie, voir cartes). Un second quartier albanais, celui de Suvi Do, se situe en bordure de l'Ibar, là encore une zone périurbaine traditionnellement albanaise, avec deux mosquées qui furent épargnées par les forces serbes. Zone moins protégée que la Petite Bosnie, le quartier bénéficie de deux accès au sud. La zone marquée des "Trois Tours " fut aussi repeuplée par des familles albanaises sous l'impulsion de la KFOR à son arrivée, dans une zone peu favorable à leur relogement : le quartier du 7 Shtatori/7Septembar, dont les immeubles sont à peuplement serbe et où se trouvent les gardiens du pont, une milice serbe surveillant les principaux axes de circulation auprès du débit de boisson la Dolce Vita, leur quartier général. Afin de faciliter les transits entre le nord et le sud de la ville, et éviter que les Albanais n'aient à " rencontrer " leurs voisins serbes, la KFOR a construit un pont et l'a fortifié. La zone juste au nord de Suvi Do, le quartier Vitakova Česma/Kroni i Vitakut était une zone mixte, elle aussi. Lors de la guerre, les Albanais quittèrent le quartier et leurs maisons furent incendiées, puis ils revinrent à la faveur de la protection que leur a accordé l'OTAN dans cette zone. Kodra e Minatorëve est un autre quartier du nord de la

${ }^{20}$ Interviews à Paris et Mitrovica, 2001 et 2002 . Voir aussi ICG, " Kosovo's Linchpin : Overcoming division in Mitrovica ", Balkan report, (96), 31st May 2000. 
ville, situé en surplomb de l'hôpital. Un contrôle militaire barre l'accès aux personnes qui voudraient s'y rendre car c'est aussi un quartier mixte, peuplé à $60 \%$ d'Albanais mais aussi de populations roms, bosniaques et de Serbes.

Globalement, le nord de Mitrovica/Mitrovicë a subi ces dernières années des mouvements de population importants. En effet, les forces de sécurité serbes et yougoslaves ont fait fuir les populations albanaises, dans le cadre plus général de l'expulsion des populations albanaises hors du Kosovo durant les frappes de l'OTAN au printemps 1999. Ces populations, dont un nombre non-négligeable était revenu au nord de la ville en juin 1999 suite à l'arrivée de la KFOR, ont été ré-expulsées par la population serbe en septembre 1999 et au début de l'année 2000 , suite à des manifestations et des intimidations violentes ${ }^{21}$. Le mouvement inverse a ensuite été observé, puisque des familles albanaises ont été relogées au nord, dans ces quartiers mixtes que nous venons d'énumérer, sous la pression et la protection de la communauté internationale. Ils ne peuvent cependant circuler sans entrave ni danger dans l'ensemble de l'espace urbain, et bénéficient d'accès au sud de la ville (voir carte 2), de sorte qu'une cohérence territoriale existe entre une zone sud à peuplement quasi-exclusivement albanais et les zones nord-ouest et de la Petite Bosnie, à peuplement mixte.

L'ambiance dans la partie nord de la ville est donc extrêmement tendue, comme peuvent en attester les Check-Points mis en place par la KFOR. Bien que la stratégie de l'OTAN, la " non fixing strategy " initiée en 2002, consiste à éliminer les points de contrôles fixes au profit de patrouilles, rendant moins perceptible la militarisation de l'espace urbain, il reste qu'un ensemble d'éléments contribue à ce que l'atmosphère ne soit pas sereine; entre autres la présence persistante des milices serbes gardant le pont, un réseau de fils de fer barbelés, et la perception diffuse que la situation peut se dégrader très vite. Qui plus est, l'impression qui se dégage d'une promenade à Mitrovica/Mitrovicë nord reste celle d'une vie assez miséreuse. Si l'on compare avec le sud de la ville, les marchés ne sont pas aussi fournis, les prix sont eux-même bien moins élevés, et les voitures que l'on aperçoit ne sont pas non plus les voitures allemandes dans lesquelles on peut voyager de l'autre côté de l'Ibar.

\section{L'échelon de la ville : les fractures et polarités de l'espace urbain}

Si l'on fait abstraction de la diversité du peuplement à l'échelle des quartiers, l'espace urbain se structure au niveau d'analyse supérieur selon une logique de fractionnement binaire. En effet, dans les déclarations officielles des dirigeants de la MINUK, comme dans la logique de division territoriale du pouvoir politique, la ville est traversée par une fracture qui épouse partiellement le cours de l'Ibar.

${ }^{21}$ Voir ICG, « Kosovo's Linchpin : Overcoming division in Mitrovica ", (art.cit.) ; IWPR, 1999. 
Carte 1 : Situation de Mitrovica à l'arrivée de la KFOR, été 1999.

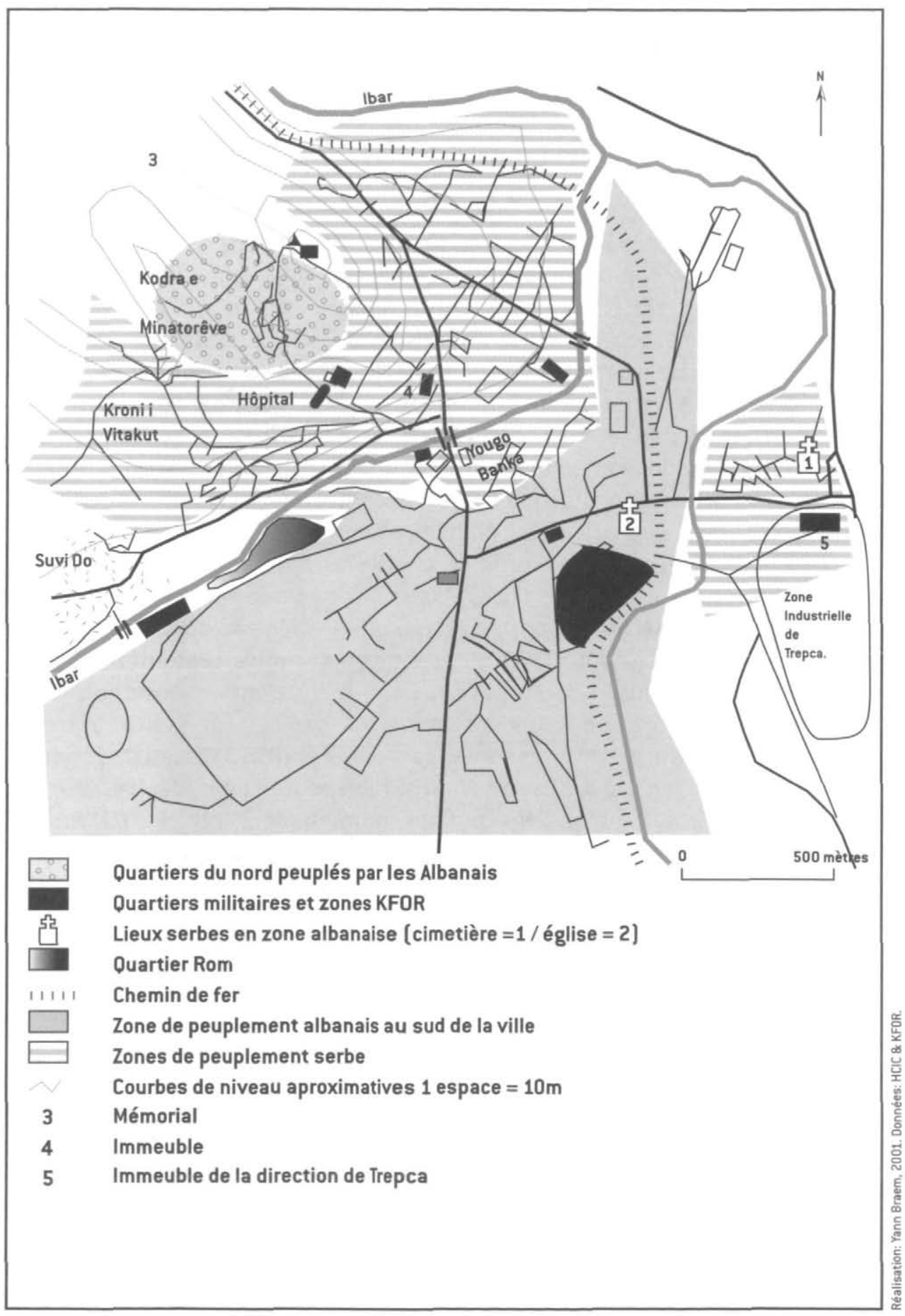


Carte 2 : Situation de Mitrovica en février-mars 2001.

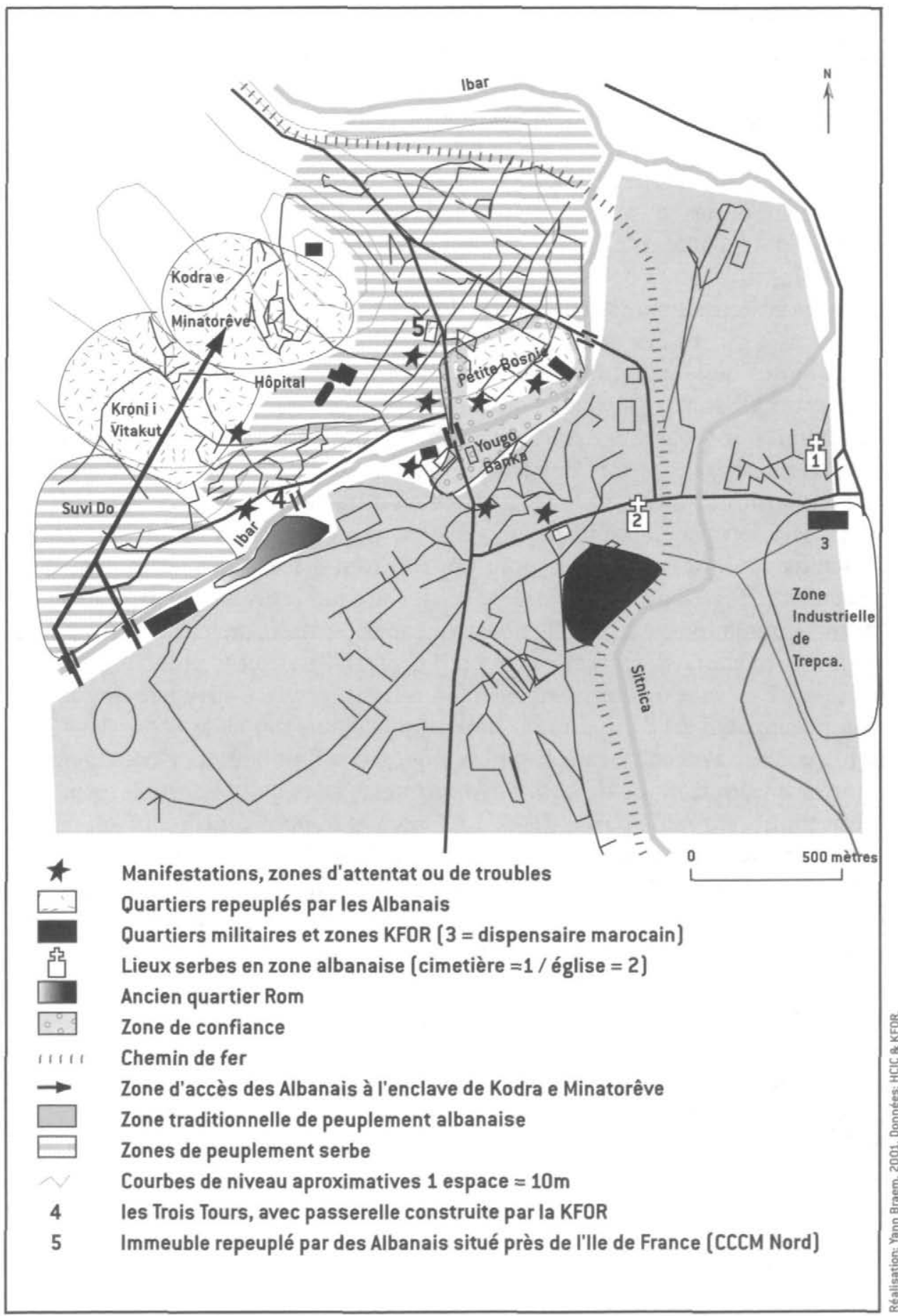


De ce point de vue, les discours, aussi bien de la communauté internationale que ceux des populations locales parlent bien du nord et du sud comme de deux espaces différenciés, fractionnant l'espace urbain le long de la rivière. La rivière a donc un statut particulier, et, au-dessus, le pont principal situé à côté de la Yougo-Banka est l'objet d'une surveillance militaire et paramilitaire imposante. Les fortifications de la KFOR sont ainsi les plus importantes autours de cet axe : l'accès au pont principal est étroitement surveillé, les éventuelles personnes le traversant sont soumises à contrôle d'identité et fouille en règle par les soldats de la KFOR. C'est aussi autour de ce pont que se concentraient les dispositifs de surveillance paramilitaires serbes (ceux que l'on appelle traditionnellement les "Bridge-Watchers ") et albanais ${ }^{22}$, qui opéraient de temps à autres des contrôles d'identité illégaux. Enfin, il est à noter que nombre des incidents qui surviennent à Mitrovica/Mitrovicë entre les communautés albanaises et serbes ont lieu le plus souvent autours de ce pont, et à proximité de l'Ibar. L'axe routier central du côté nord est ainsi l'endroit où se réunissent les Serbes lors des manifestations. De même, les affrontements entre manifestants albanais et forces de sécurité de la KFOR survenus lors des manifestations de septembre 1999 ont eu pour théâtre le pont central, les Albanais voulant traverser le pont pour affronter les Serbes et mettre fin à l'expulsion des Albanais du nord de la ville. De fait les manifestants auraient pu traverser la rivière à pied (le niveau d'eau le permet), ou par d'autres ponts, mais ils ont choisi de passer par le pont central, l'endroit le plus fortifié de la ville, situé en face des immeubles de la MINUK et placé sur l'axe de circulation principal. Il est d'ailleurs révélateur que le quartier de la Petite Bosnie en bordure de rivière soit beaucoup plus sujet à des flambées de violence que le quartier multiethnique de Kodra e Minatorevë, alors que ce dernier est nettement moins surveillé par la KFOR. La rivière et le pont central qui l'enjambe sont donc des éléments cruciaux d'une représentation de l'espace urbain, la conflictualité du lieu étant davantage liée à la symbolique qui s'y attache, qu'à l'aspect purement tactique de la volonté de traverser la rivière. Dans les moments de violence, l'important serait donc de traverser le pont, de l'occuper, comme symbole de victoire, plus que de traverser la rivière en vue de provoquer un choc frontal.

Cette perception d'une polarisation de l'espace urbain autour d'un axe suivant l'Ibar est renforcée par l'existence de centres de pouvoir politique municipaux très distincts. Au sud, et comme sur l'ensemble de l'espace kosovar, la MINUK possède la haute-main sur les décisions politiques, alors même qu'une assemblée municipale composée d'élus locaux désignés au suffrage universel cogère la ville avec les organes de la communauté internationale. Cette assemblée

\footnotetext{
${ }^{22}$ Ces personnes chargées de surveiller le pont côté albanais ont disparu durant l'année 2002, alors que les Bridge-Watchers, eux, restent en place.
} 
municipale est exclusivement composée de membres de la communauté albanaise, la population serbe ayant boycotté les différents scrutins, par conviction quelque peu " forcée ", les miliciens serbes empêchant les électeurs de se rendre aux urnes pour les élections municipales de 2000 et de 2002 et les élections générales de 2001 . On pourra noter d'ailleurs que la tendance électorale de Mitrovica/Mitrovicë, c'est-à-dire celle des Albanais de la ville, voit le renforcement du Partia Demokratike e Kosovës (Parti Démocratique du Kosovo, PDK, dirigé par Hashim Thaçi) de près de 10 points et une baisse de la Lidhja Demokratike e Kosovës (Ligue Démocratique du Kosovo, LDK, dirigée par Ibrahim Rugova) ${ }^{23}$ de plus de 13 points. Ce recul d'un parti plus modéré, dont le leader est perçu comme très éloigné de la population, et le renforcement d'un parti plus radical ${ }^{24}$, issu directement de la guérilla et partisan d'une indépendance inconditionnelle, peut apparaître comme un signe de lassitude des populations albanaises vis-à-vis des organes de la communauté internationale, exerçant la réalité du pouvoir au Kosovo, ou de radicalisation vis-à-vis de voisins serbes perçus comme des trublions. Au final, les lieux du pouvoir politique au sud de la ville restent les immeubles administratifs du quartier de la YougoBanka, bureaux de la MINUK régional et municipal, ceux de l'OSCE toute proche, les bâtiments du Kosovo Police Service ainsi que l'immeuble de la Municipalité situé en face du quartier de la Yougo-Banka. Il faudrait leur ajouter bien sûr le quartier général de la brigade multi-nationale nord situé au sud de l'église orthodoxe, responsable de toute l'Aire de Responsabilité confiée aux armées françaises.

Ces centres de pouvoir localisés au sud de la ville n'ont cependant qu'une influence limitée sur l'ensemble de l'espace urbain, et notamment le nord. C'est d'ailleurs pour cela que l'on peut ici parler de fracture géopolitique. Si la KFOR possède les moyens de contrôler militairement l'espace de Mitrovica/Mitrovicë, et d'empêcher que la violence ne se manifeste de manière trop durable ou trop fréquente, la MINUK, elle, n'est pas en mesure de gérer cette partie de la ville. En avril 2002, par exemple, suite à la tentative

\footnotetext{
${ }^{23}$ Lors des élections de 2000 (municipales), 2001 (législatives) et 2002 (municipales), la LDK, parti qui avait prôné la résistance pacifique lors de la décennie 1990, a vu son score baisser de $67,3 \%$ des suffrages exprimés à $56,49 \%$ puis $53,48 \%$. Le PDK, parti plus nationaliste, héritier direct de l'UÇK voit, lui, son influence considérablement renforcée, passant de 24,1 à 28,08 puis 34,2 \% des suffrages exprimés. L'AAK (Aleanca për Ardhemërinë e Kosovës, Alliance pour l'Avenir du Kosovo) dirigée par Ramush Haradinaj et elle-même issue de la guérilla de l'UÇK, mais prônant des mesures moins radicales que le PDK en termes de réformes économiques (privatisations acceptées) et de dialogue avec les autorités internationales (notamment sur les modalités d'accession à l'indépendance), reste relativement stable, avec respectivement 3,1; 6,32 et 5,71\% des suffrages exprimés dans la municipalité de Mitrovica. Résultats certifiés par l'OSCE, voir http://www.osce.org/kosovo/elections.
}

24 Il semble en effet que le recul de la LDK profite directement au PDK, le parti " arbitre ", l'AAK, restant en stagnation, et la correspondance entre les pertes de la LDK et les gains du PDK étant numériquement révélatrice d'un report d'une partie de l'électorat du parti de $M$. Rugova vers celui de $M$. Thaçi. 
d'arrestation d'un des membres de la milice serbe par la police de la MINUK, des manifestations très violentes destinées à protéger le Bridge-Watcher ont menacé directement les éléments de la MINUK, les composantes civiles de la communauté internationale étant de facto exclues de la partie nord de la ville. L'absence de participation de la communauté serbe n'est, dans cette mesure, que le symptôme, la face cachée, d'un système politique et administratif parallèle bien connu de tous les acteurs locaux et internationaux ${ }^{25}$. La question du monopole de la violence légitime, signe d'une " souveraineté " d'un acteur politique déterminé, n'est donc pas réglée au nord de Mitrovica/Mitrovicë, nous y reviendrons. De même, un autre symptôme de la division de la ville peut être envisagé, celui de la division monétaire, puisque les monnaies en circulation au nord de la ville sont l'euro, comme valeur monétaire refuge, et le dinar yougoslave comme monnaie d'échange courant, considérée comme la monnaie officielle par les habitants du nord, mais qui n'est pas acceptée au sud de la ville. La division de la ville se manifeste donc de manière multiple, et, entre le nord et le sud, les différences sont importantes, palpables aux niveaux économiques, politiques, et, bien sûr, ethnique. Il existe de ce fait un système politique serbe alternatif de celui installé par la MINUK, organisé autour de l'hôpital de Mitrovica/Mitrovicë : un lieu qui connut des " péripéties " révélatrices de l'histoire de la ville à bien des égards. Ce système politique possède ses relais administratifs, avec une mairie, des services administratifs, des fonctionnaires payés par le gouvernement serbe de Belgrade, et des forces de " police ", les BridgeWatchers, eux aussi rémunérés par la République de Serbie.

Le pouvoir politique à Mitrovica/Mitrovicë est de ce fait "partagé n entre plusieurs systèmes politiques, et le pouvoir au nord de la ville lui-même divisé entre plusieurs personnalités, sans toutefois que les responsabilités spécifiques ou l'équilibre des pouvoirs ne puisse être véritablement évalué. Parmi ses hommes se trouvent Marko Jakšić, Vladimir Adžić et Milan Ivanovićc ${ }^{26}$, respectivement directeur du centre de santé, chef du service orthopédie et directeur de l'hôpital. Ils seraient, mais surtout Marko Jakšić ${ }^{27}$, les dirigeants des services paramilitaires de sécurité qui quadrillent le nord de Mitrovica/Mitrovicë. Ces trois hommes sont effectivement les seuls à être restés au Kosovo parmi les membres d'une élite locale pouvant " prétendre " au

\footnotetext{
25 ICG, "Kosovo's Linchpin : Overcoming division in Mitrovica ", (art.cit.) ; ICG, "UNMIK's Kosovo Albatros : tackling division in Mitrovica ", (art.cit.) ; European Stability Initiative (ESI), People or territory? A proposal for Mitrovica, February 2004 (texte disponible sur http://www.esiweb.org).

${ }^{26}$ Milan Ivanović fut arrêté en 2002 par les services de police de la MINUK sous l'inculpation de crime de guerre supposés commis durant le conflit de 1999. Il fut relâché immédiatement après avoir été arrêté, ce qui provoqua la colère et l'incompréhension d'un certain nombre d'Albanais de Mitrovica.
}

${ }^{27}$ Interview avec François Crémieux, ancien directeur de l'hôpital, Paris 2003, avec un conseiller politique du Général Commandant la BMN nord. Voir aussi ICG, op. cit. 2000 et 2002. 
leadership local ${ }^{28}$, dans la crise du lien politique d'après-guerre. L'hôpital est ainsi lieu de pouvoir par ses occupants, puisque le Kosovo n'est plus géré par la République de Serbie et que la MINUK est perçue comme un acteur pro-albanais. Il l'est également dans la mesure où le pouvoir des médecins est " reconnu "; les visites régulières qu'effectuent les militaires de la KFOR, notamment les médecins militaires, mais aussi certains membres de la communauté internationale étant de nature à renforcer l'idée d'une centralisation du pouvoir politique autour de l'édifice hospitalier. Lieu politique, principal employeur du nord de la ville, l'hôpital est donc au centre d'enjeux de pouvoir politique et économique ayant débuté en 1999 à l'arrivée de la KFOR et de la MINUK. Lorsque la communauté internationale fut chargée de gérer l'hôpital, ses personnels hissèrent le drapeau de la MINUK, avant de faire travailler ensemble Serbes et Albanais pour soigner des membres de toutes les communautés. L'hôpital était désigné d'emblée comme symbole de la multi-ethnicité, revendiquée par la communauté internationale ; une multi-ethnicité mise à mal lors des événements de septembre 1999, date de l'expulsion des travailleurs albanais de l'hôpital, correspondant au début de l'expulsion des Albanais du nord de la ville, et, ce faisant, la reprise du contrôle de l'hôpital par ses dirigeants actuels. Enfin, ce bref aperçu du paysage politique serbe de Mitrovica/Mitrovicë ne serait pas exhaustif si l'on omettait Oliver Ivanović, lui aussi soupçonné de diriger une partie des miliciens, et qui est devenu un acteur local incontournable, avant d'être élu en 2001 député à l'Assemblée du Kosovo et membre de la Présidence de cette dernière, nous y reviendront.

\section{LA DIVISION DE MITROVICA/MITROVICË, UNE REPRÉSENTATION GEOPOLITIOUE}

La division de Mitrovica/Mitrovicë, pour autant qu'elle soit effective dans la différenciation des centres de pouvoir, est aussi une représentation géopolitique, et, à ce titre, sert un certain nombre d'intérêts, et notamment ceux des

\footnotetext{
${ }^{28} \mathrm{Dans}$ les recompositions politiques que la ville a pu connaître dans la période de post-conflit, le rôle du médecin, de par sa position professionnelle (voir ici la différenciation entre un métier et une profession opérée par C. Dubar, in Dubar (Claude), La socialisation, Construction des identités sociales et professionnelles, Paris : Armand Colin, 1998) peut être envisagé comme centrale. La position particulière des acteurs serbes de l'hôpital, mais aussi du chirurgien Bajram Rexhepi, aujourd'hui Premier ministre du Kosovo, anciennement Maire par intérim de Mitrovica avant les élections municipales de 2000, peut être partiellement expliquée par leur position socioprofessionnelle, au delà de leur rôle important joué en tant qu'acteurs militaires dans la phase de conflit. De la même manière, Oliver Ivanović, un autre leader serbe de Mitrovica, est un ancien animateur de quartier, et occupait lui aussi une position socio-professionnelle spécifique, propre à lui * offrir n une clientèle politique importante.
} 
élites politiques serbes ${ }^{29}$. Effectivement, l'espace urbain n'est pas réellement divisé en deux parties, de sorte qu'il y aurait deux ensembles homogènes, avec des centres identifiés. La description des quartiers de Mitrovica/Mitrovicë nous montre au contraire une pluralité d'espaces dont le contrôle effectif est assumé par différents organes politiques et/ou sécuritaires. Le sud de la ville, ethniquement et politiquement relativement homogène, est sous le contrôle effectif des forces de sécurité dépendant des Nations unies : la police de la MINUK, le Kosovo Police Service, qui est l'organe de police locale formé par l'OSCE, et la KFOR dont le commandement est partagé entre le quartier général de la KFOR situé à Priština/Prishtinë, et la brigade multi-nationale nord qui dépend aussi de l'état-major des armées à Paris. La Zone de Confiance, qui s'étend du quartier de la Yougo-Banka jusqu'aux abords de la Petite Bosnie, est une zone à forte composante de sécurité militaire, et dépend dans une moindre mesure du contrôle de la police de la MINUK. Les zones de peuplement serbe en dehors de la Zone de Confiance sont, elles, soumises au contrôle de sécurité effectif de la KFOR, des milices serbes, et dans une moindre mesure de la MINUK Police, sous réserve d'acceptation par les miliciens serbes. Enfin, les zones de peuplement mixte ou albanais dans le nord de Mitrovica/Mitrovicë sont sous la responsabilité de la KFOR, de la MINUK Police, là encore sous réserve d'acceptation, et parfois de quelques éléments démobilisés mais toujours actifs de l'ex-UÇK. On obtient donc, en examinant d'un peu plus près l'exercice effectif de fonction de sécurité, quatre types d'ensembles géographiques distincts, ce qui contraste avec la perception d'une ville coupée en deux parties, tant dans l'exercice des fonctions sécuritaires que dans la diversité constatée de la répartition ethnique du peuplement entre les communautés serbes, albanaises, roms ou bosniaques.

C'est lorsque l'on examine la question de l'exercice du monopole de la violence légitime selon le schémas wébérien, c'est-à-dire la violence acceptée par la population, que la perspective change, et que la représentation géopolitique de la partition de la ville révèle des enjeux de pouvoir. Dans cette optique, la configuration spatiale est toute autre, et nécessite d'envisager les représentations des acteurs locaux. Si les forces de la police de la MINUK ne sont pas acceptées au nord de la ville, dans les espaces de peuplement serbe, ceci est en effet en partie dû à des représentations géopolitiques actives et mobilisatrices, qui font que l'exercice de la violence par ces forces de sécurité n'est pas acceptable par la population, qui se rebelle aussitôt, encadrée par les milices le plus souvent fortement armées. La représentation que se font les Serbes de Mitrovica/Mitrovicë nord, de "leur " espace urbain, est celle d'un espace front, une zone tampon entre une population albanaise qui voudrait les expulser du 
Kosovo ${ }^{30}$, perception que les événements de mars 2004 contribueront à renforcer, et un espace peuplé majoritairement de Serbes, sur les trois communes du nord du Kosovo. La militarisation de l'espace fluvial, celle opérée par les forces de l'OTAN mais aussi la présence des milices, est aussi signifiante qu'une frontière, passer le pont central revenant par bien des aspects à passer des postes de douanes.

Cette représentation d'une cohérence territoriale du peuplement serbe, appuyée sur une appropriation du territoire conçue sur le mode de la " défensive ", se traduit d'ailleurs dans une différenciation politique entre les espaces du nord du Kosovo et les enclaves serbes dans les zones à peuplement majoritaire albanais. En effet, si l'on observe les résultats aux élections municipales de $\mathbf{2 0 0 2}$, on constate que la coalition Povratak représente le mouvement politique le plus souvent majoritaire dans les enclaves serbes ${ }^{31}$. A contrario, on peut voir que les premières forces politiques des communes de Zvečan/Zveçan, de Zubin Potok et Leposavić/Leposaviq sont le DSS, le parti de Vojislav Koštunica et le Conseil national serbe du nord du Kosovo ${ }^{32}$. Les deux types de partis, dont les résultats diffèrent nettement en fonction de leur origine géographique, représentent également des visions politiques radicalement différentes, personnifiées dans l'opposition entre Rada Trajković33 et Marko Jakšić,

$3^{\circ}$ On pourra évoquer ici les trois "querelles " opposant les Serbes et les Albanais sur le territoire du Kosovo, relevées par M. Roux (Roux (Michel), op.cit.) et exacerbées par un sous-développement général à l'ex-Yougoslavie du sud (voir Clayer (Nathalie), "La société du Kosovo au cœur de dix années de crises ", in Yerasimos (Stéphane), éd., Le retour des Balkans (1991-2001), Paris : Autrement, 2002) : la querelle du premier occupant, celle des équilibres démographiques et celle des migrations régionales. En effet, les deux populations revendiquent l'antériorité historique de l'occupation du territoire qui agit comme processus de légitimation de leurs velléités de contrôle (voir Guillorel (Hervé), Michels (Patrick), " Continuité territoriale, continuité nationale : l'exemple yougoslave ", Balkanologie, 1 (1), juillet 1997 ; ainsi que Roy (Olivier), La nouvelle Asie centrale ou la fabrication des Nations, Paris : Seuil, 1997 ; sur la " fabrication " des nations en Asie centrale par le système soviétique et l'utilisation des sciences humaines et sociales comme instrument de légitimation nationale). Ensuite les Serbes accusent les Albanais de mener à dessein une stratégie de fort accroissement démographique afin de bénéficier du déséquilibre entre les masses de population (à peu près $90 \%$ d’Albanais et $10 \%$ de Serbes en 1991). Enfin, les premiers accusent les Albanais d'Albanie d'avoir migré au Kosovo sous l'occupation albanaise durant la Seconde Guerre mondiale, alors que les Serbes ont tendanciellement plus migré vers la Serbie à la recherche d'un emploi au cours de la seconde moitié du XX ${ }^{e}$ siècle.

${ }^{31}$ On constate en effet que dans les 15 municipalités où des Serbes sont représentés (en dehors des trois municipalités du nord de la Province), la coalition Povratak a obtenu 19 sièges aux élections municipales de 2002, le DSS n'en ayant compté que 9 (Résultats certifiés OSCE).

${ }^{32}$ Dans la commune de Zvečan/Zveçan : le DSS a obtenu en $200230,04 \%$ des suffrages exprimés, le CNS $34,54 \%$ et Povratak $11,73 \%$. Dans celle de Zubin Potok : DSS, $45,88 \%$; le CNS ne s'est pas présenté ; Povratak, $3,70 \%$. Dans celle de Leposavić/Leposaviq : DSS, $25 \%$; SNV, 18,07 \% ; Povratak, 13,36 \% (Résultats certifiés de l'OSCE).

33 Représentante de la coalition Povratak, symbole d'un courant libéral serbe ayant ses appuis en Serbie auprès du défunt Premier ministre Zoran Đinđić et dont le programme politique affirme la nécessité de couper les liens avec Belgrade. Voir Matveeva (Anna), Paes (Wolf-Christian), The Kosovo Serbs : An ethnic minority between collaboration and defiance, Postdam : The Friedrich Naumann Foundation, 2003. 
entre une acceptation de la communauté internationale et l'affirmation de la souveraineté toute puissante de Belgrade sur l'ensemble du Kosovo. En effet, la différenciation entre les enclaves et l'espace serbe au nord du Kosovo s'appuie en partie sur le rôle qui est attendu de Belgrade, et sur la perception de la communauté internationale. Cette représentation de cohérence territoriale s'appuie en outre sur les plans de redécoupages territoriaux proposés par Belgrade 34 et l'European Stability Initiative (ESI, 2004), proposant l'unification de la municipalité de Zvečan/Zveçan et le redécoupage de la municipalité de Mitrovica/Mitrovicë, entérinant la division de la ville le long de l'Ibar. Si, en effet, les deux municipalités n'en formaient qu'une en 1986, les plans de partage qui sont proposés diffèrent cependant de cet espace politico-administratif, puisqu'ils supposent la division selon l'axe de l'Ibar, ce qui n'était pas le cas dans les années 1980 , et entérine cette représentation géopolitique d'une cohérence du peuplement serbe en l'officialisant à travers un organe institutionnel.

Le ressenti d'un espace front entre des bassins de peuplement serbe et albanais fortement antagonistes ne correspond pas à la vision d'un front statique. Bien au contraire, l'une des représentations géopolitiques les plus actives et les plus mobilisatrices au sein de la population serbe est illustrée par la carte 3 et plus largement l'ensemble de nos trois cartes. Cette carte montre la vision d'encerclement qu'ont les Serbes de Mitrovica/Mitrovicë, et qui est étroitement corrélée à l'activisme de la communauté internationale en faveur du retour des populations albanaises au nord de l'Ibar. D'une part, les différents quartiers repeuplés par les Albanais au nord-ouest de la ville sont perçus comme des avancées, des percées mettant en péril le contrôle serbe de cet espace urbain car elles les mettraient en situation de minorité, et remettrait en cause leur présence même 35 . D'autre part, la question de l'extension de la Zone de Confiance, posée au début de l'année 2001 par la MINUK en vue de permettre aux Albanais d'accéder à l'hôpital et de reprendre certaines de leurs habitations le long de cet axe, a été vécue comme une double menace. Elle supposait en effet une perte de contrôle de l'élite serbe de l'hôpital, mais aussi un retour des Albanais dans une zone d'où ils avaient été exclus quelques mois

\footnotetext{
34 L'une des propositions de découpage du Kosovo, émise par Branislav Krstić (Roux (Michel), art.cit.) et sur laquelle se serait appuyée Nebojša Čović (ICG, "UNMIK's Kosovo Albatros : tackling division in Mitrovica ", art.cit.) serait effectivement soit de rẻunir les municipalités du nord du Kosovo avec Mitrovica nord, dans une entité politico-administrative serbe, soit de réunifier les municipalités de Zvečan/Zveçan et de Mitrovica/Mitrovicë, comme c'était déjà le cas en 1986 (ESI, op.cit.).

35 D. Jovic (Jovic (Dejan), "Fear of becoming a minority as a motivator of conflict in the Former Yougoslavia ", Balkanologie, 5 (1-2), décembre 2001) a récemment montré que la perspective de se trouver en situation de minorité était perçue dans l'espace ex-yougoslave comme un danger, remettant en cause l'idèe même de rester sur le territoire concerné, et accroissant les conflits. De ce fait, la mécanique du conflit place le peuplement au centre de ses enjeux, l'objectif étant de maintenir le peuplement majoritaire et donc d'agir sur les populations " adverses ".
} 
Carte 3 : L'extension de la zone de confiance.

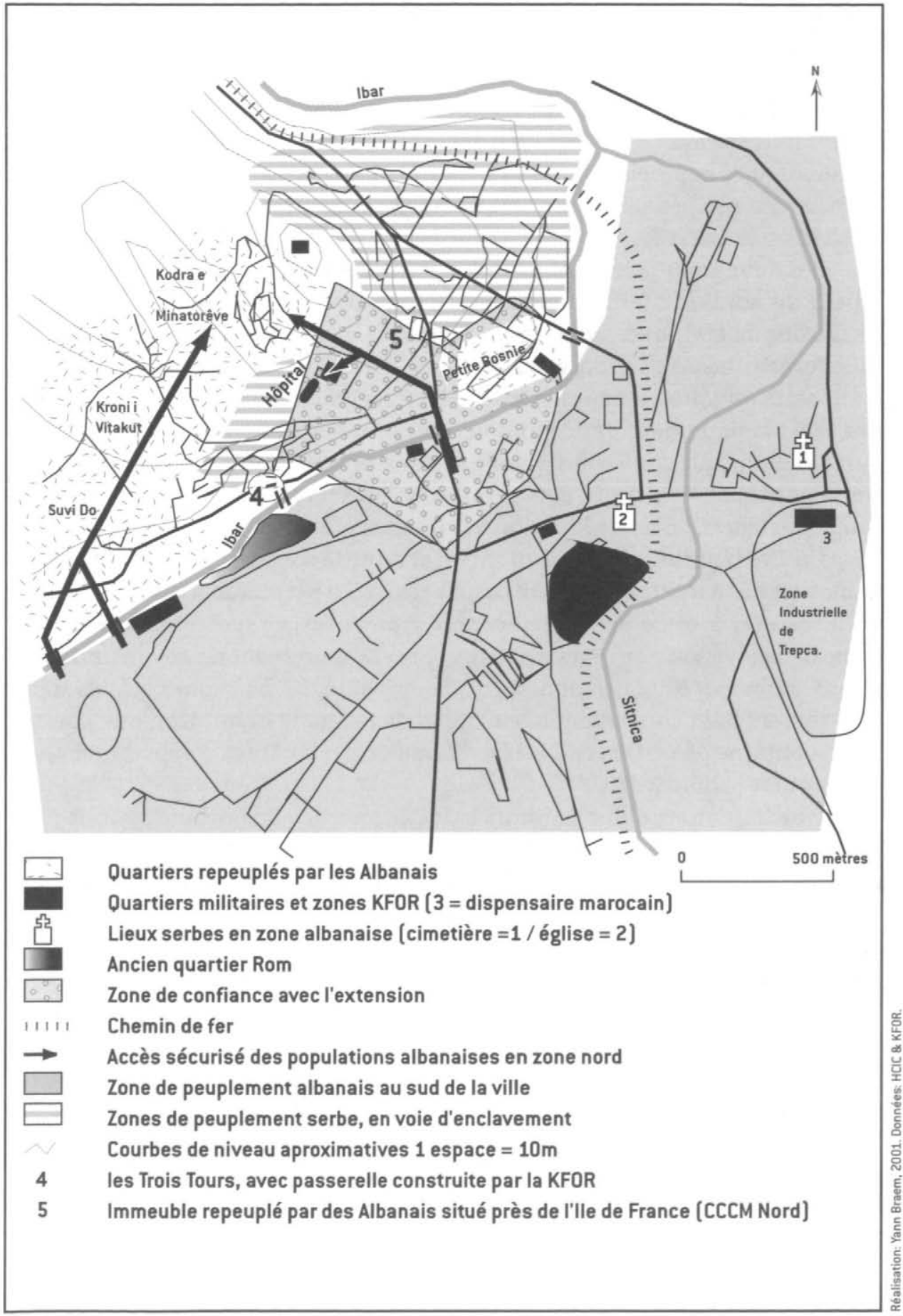


auparavant. Qui plus est, elle aurait fourni une seconde voie d'accès à l'enclave multiethnique de Kodra e Minatorëve, et donc un espace d'action supplémentaire pour les extrémistes albanais qui n'auraient alors pas manqué, se disaient les Serbes ${ }^{36}$, de les harceler pour les faire partir et prendre le contrôle de cette partie de la ville. Coupée de ses " bases arrières ", une partie de la population auraient donc été mise en situation d'enclave, encerclée par les Albanais. Plus généralement, la superposition des trois cartes présentées nous permet de cerner le recul qui est perçu par les Serbes sur la zone front depuis l'arrivée de la MINUK et de la KFOR.

Cette dynamique territoriale est ressentie, dans le cas des relogements albanais au nord de la ville, mais encore plus dans l'hypothèse d'une extension de la Zone de Confiance, comme un ensemble de reculs sur une zone de front, directement liés à l'action et la présence de la communauté internationale. Dans cette optique, l'acteur le plus légitime, celui dont on n'a le moins à craindre, n'est logiquement pas celui dont on est convaincu qu'il a œuvré à ces reculades. Dans une zone conflictuelle comme celle de Mitrovica/Mitrovicë, l'acteur politique légitime est celui qui protège de l'ennemi, donc les Bridge-watchers, l'élite de l'hôpital qui les dirige, et le gouvernement serbe qui les paie. L'autre acteur légitime, dont on accepte le contrôle, reste la KFOR, dans la mesure où la militarisation de l'espace fluvial a permis de mettre fin à ce qui était ressenti comme une forte avancée albanaise. La représentation géopolitique de la division nord/sud, appuyée sur celle d'une cohérence territoriale du peuplement serbe aboutit donc à ce que les stratégies politiques locales soient étroitement liées à une demande d'appui de Belgrade, contrairement à la stratégie politique de certaines zones enclavées, dont les élites s'appuient d'abord et avant tout sur la MINUK.

Pour autant les élites politiques de Mitrovica/Mitrovicë ne se sont pas " enfermées " dans une position d'opposition systématique à la communauté internationale. Oliver Ivanović occupe dans cet échiquier politique local une place à part, ayant pris le parti de s'appuyer sur Belgrade comme sur les différents acteurs internationaux dès l'arrivée de la MINUK et de la KFOR ${ }^{37}$, aboutissant ainsi à être nommé à la présidence du Parlement à la suite de son élection en tant que député au Parlement au sein de la coalition Povratak. Les autres leaders de Mitrovica/Mitrovicë suivent des stratégies politiques beaucoup plus ambiguës. Appelant au boycott des élections à Mitrovica/Mitrovicë, et donnant instructions aux milices d'empêcher la participation des Serbes au vote, Marko Jakšić, Vladimir Adžić et Milan Ivanović n'en furent pas moins

${ }^{36}$ Entretiens menés à l'hôpital de Mitrovica et au Quartier Général de la Brigade Multi-Nationale nord en 2001.

37 Il ne s'est d'ailleurs pas opposé à la tenue d'élections législative en 2001, et pour cause, il était candidat. Il s'était par contre prononcé en faveur d'un boycott des élections municipales de 2000. 
élus au cours de ces scrutins dans d'autres municipalités. Vladimir Adžić et Milan Ivanović siègent ainsi au conseil municipal de Zvečan/Zveçan sous les étiquettes du SNC et du DSS, alors que Marko Jakšić est, lui, élu municipal à Zubin Potok sous l'étiquette du DSS, dont il est d'ailleurs vice-président. Se ménageant des positions de pouvoir dans d'autres municipalités leur permettant d'être des interlocuteurs politiques vis-à-vis de la communauté internationale, et se recycler en cas de baisse de leur influence à Mitrovica/Mitrovicë, ces acteurs furent dans le même temps les principaux obstacles à l'établissement de la municipalité de la MINUK au nord de l'Ibar, et au déroulement d'un processus électif conjoint entre le nord et le sud de la ville. Le blocage de la situation politique, s'appuyant sur la peur géopolitique de l'encerclement et de l'invasion albanaise, leur permet ainsi de se ménager un monopole des positions de pouvoir au nord de la ville, sans concurrence de la part de communauté internationale ou d'autres leaders. Il leur permet par ailleurs de conserver la position socio-économique qui fut à l'origine de leur influence politique et leur fournit aujourd'hui des ressources économiques non négligeables (salaires de l'hôpital payés par Belgrade, contrôle d'activités illégales à travers la milice), qui pourraient être remises en cause par le passage de cet hôpital sous la direction de la MINUK et la reprise d'une politique de recrutement multiethnique. Ces acteurs ont donc réussi à démultiplier les " points d'ancrage " fournissant des ressources politiques. D'une part, leurs liens avec le parti DSS au pouvoir à Belgrade leur fournit une légitimité politique importante, celle de la " mère patrie n-mais aussi celle du bailleur de fonds - tout en leur laissant une marge de manœuvre importante au niveau local. Effectivement, alors même que le gouvernement serbe et la présidence yougoslave appelait dès 2001 à la participation aux processus électifs, ces acteurs locaux empêchaient la participation serbe au scrutin à Mitrovica/Mitrovicë nord, s'appuyant sur d'autres ressources plus localisées, d'ordre socio-politique (position socioprofessionnelle de l'hôpital, appui sur la représentation de la menace albanaise) ou économique (la fin annoncée de l'appui économique de Belgrade aux milices serbes a pu être compensée par le contrôle sur les activités illégales : prostitution, trafic de drogue). D'autre part, cette position de monopole politique les a " imposés " comme interlocuteurs de la communauté internationale, et particulièrement de la KFOR pour la gestion au quotidien de la sécurité de la ville, tout en restant assurés d'une légitimité élective dans d'autre municipalités.

Du côté albanais, la représentation géopolitique d'une division de la ville est aussi très active. L'exercice du monopole de la violence légitime est reconnu comme étant attribut de la communauté internationale au travers de ses différentes composantes, la KFOR, la MINUK Police et les forces du Kosovo Police Service. Cependant, l'absence d'unicité du contrôle de sécurité sur l'ensemble de la ville laisse les Albanais très amers face à ce qu'ils perçoivent comme une survivance de l'ancien ordre serbe, mais également comme une remise en 
cause de l'unité du territoire du Kosovo, dont il désirent obtenir l'indépendance. Effectivement, ils décrivent bien souvent les miliciens, ou encore certains leaders de Mitrovica/Mitrovicë nord, comme d'anciens criminels de guerre s'étant recyclés dans le contrôle de la ville, ou comme des soldats de l'armée yougoslave revenus au Kosovo en civil, suivant les informations publiées au niveau international ${ }^{38}$. L'absence de contrôle effectif ou légitime de la part de la MINUK au nord de la ville est ainsi source de frustration politique, dans la mesure où elle induit une division de fait du territoire, mais aussi source de colère dans la mesure où le contrôle de Belgrade est interprété comme niant la victoire de l'UÇK et de l'OTAN sur les forces serbes.

De ce point de vue, la représentation albanaise du territoire kosovar, c'està-dire du territoire qui leur revient en vertu de la victoire qu'ils ont remporté contre les forces yougoslaves ${ }^{39}$, est calquée sur le découpage de l'entité administrative du Kosovo opéré par la Yougoslavie titiste en $1945^{40}$. Elle n'est pas susceptible d'être remaniée à la faveur de réaménagements territoriaux selon une ligne ethnique comme on pourrait le supposer. Les territoires revendiqués par Priština/Prishtinë ne sont pas les espaces de peuplement albanais actuels, auquel cas la partition entre un nord serbe et un sud albanais serait acceptable, mais l'espace administratif défini en 1945, devenu un " territoire national ". La territorialité $4^{1}$ albanaise ne s'arrête donc pas aux marges du peuplement, mais intègre d'autres composantes nationales. La question nationale albanaise, soulevée entre autres, et de manière maximaliste par Rexhep Qosja $4^{2}$, est ainsi liée d'une part à l'acceptation d'une unité des populations albanaises, peu légitime 43 lorsqu'on la pose comme une question régionale ; mais est aussi, d'autre part, étroitement soumise à la problématique des minorités non albanaises. À cet égard, les signaux envoyés par les populations albanaises sont fortement contradictoires, comme l'élection du Président du Kosovo l'a laissé paraître44. L'acceptation du processus de retour des populations serbes déplacées suite à l'établissement du protectorat par les Nations unies, est contredite régulièrement par les exactions dont sont victimes les mi-

$3^{8}$ ICG, " Kosovo's Linchpin : Overcoming division in Mitrovica ", art.cit. ; IWPR, 1999.

39 Lors des entretiens menés au Kosovo avec les Albanais, mais aussi au cours de conversations plus informelles, il apparaissait fréquemment que le retrait des forces serbes de la Province était une victoire de l'UÇK, appuyée par l'OTAN, et non une victoire de l'OTAN profitant à l'UÇK.

40 Roux (Michel), art.cit.

$4^{11}$ Roux (Michel), op.cit.

$4^{2}$ Qosja (Rexhep), La question albanaise, Paris : Fayard, 1995.

43 Clayer (Nathalie), " Être albanais dans les Balkans des années 1990 ", in Yerasimos (Stèphane), éd., op.cit.

44 En 2001, les résultats plaçant la coalition serbe Povratak au rang de troisième force politique aux élections générales a posé un dilemme aux partis albanais. Le Parlement du Kosovo devait effectivement 
norités non-albanaises. Cette contradiction ne pourrait être qu'apparente : l'acceptation des minorités ne serait qu'une façade acceptable que les élites albanaises présentent à la communauté internationale, tandis que le harcèlement empêcherait les retours effectifs. Elle pourrait aussi être liée à une véritable scission dans l'échiquier politique albanais du Kosovo, entre une élite représentée dans les institutions, favorable à un Kosovo multiethnique, et des mouvances plus extrémistes privilégiant la perspective d'un Kosovo purement albanais. Ces deux hypothèses peuvent se décliner à Mitrovica/Mitrovicë,le repeuplement albanais au nord de l'Ibar pouvant être analysé dans une optique " belliciste ", celle d'une offensive destinée à repousser les populations serbes ; ou dans une optique plus " politiquement correcte ", envisageant ces avancées du peuplement albanais comme conséquence logique du retour des réfugiés et déplacés dans leurs anciens foyers.

Pour les Albanais du Kosovo, l'intégrité territoriale de la Province constitue un attribut essentiel du projet d'indépendance. Sans préjuger de sa prégnance dans tous les cercles politiques, notamment au regard des représentations et projets géopolitiques de " Grand Kosovo " ou de "Grande Albanie ", on peut remarquer que les différents projets relatifs à un statut particulier de Mitrovica/Mitrovicë (partition de la ville et rattachement à des municipalités du nord) sont systématiquement rejetés dans la mesure où ils constitueraient les premiers pas vers une cantonisation du Kosovo, c'est-à-dire le retour d'une certaine forme de souveraineté serbe sur une partie du territoire ou, pire encore, vers une partition de la Province entre un nord serbe rattaché à la Serbie et le reste d'un Kosovo albanais indépendant. Ce dessein, que l'on prête souvent à Nebojša Čović, entérinerait la situation de division actuelle, alors que les Albanais réclament au contraire le déblocage de la situation au nord de la ville par l'unification de la municipalité, et donc l'exercice du monopole de la violence légitime sur l'ensemble de cet espace.

Les revendications albanaises sur le nord de la ville s'appuient d'ailleurs sur un certain nombre d'éléments de justification, dont on peut remarquer qu'ils appartiennent au registre classique utilisé par les organisations internationales comme critères de gouvernance démocratique. Ainsi, la municipalité de Mitrovica/Mitrovicë, tout comme les journalistes locaux, demandent régulièrement à ce que la Zone de Confiance soit élargie 45 , afin de mettre en oeuvre

élire le président de la Province, et devait donc constituer une alliance, la LDK avec le PDK ou avec la coalition Povratak. La LDK a hésité durant plusieurs mois entre une alliance avec son principal rival politique, le PDK, ou avec des Serbes, ce qui constituait un problème politique tout aussi important. Le parti albanais considéré comme le plus modéré a donc préféré s'allier avec son rival historique. La contradiction est donc importante, entre un discours d'acceptation des Serbes et un refus de gouverner avec eux.

45 Nous nous référons íci à des entretiens menés à Mitrovica/Mitrovicë en 2001 et 2002, et notamment avec Mustaffe Pllana, adjoint au Maire de la municipalité. 
la liberté de circulation, qui reste l'un des objectifs de la communauté internationale, et le retour des déplacés, des anciens habitants albanais au nord de la ville. Cette liberté de circulation, de même que l'extension de l'autorité de la police civile au nord de la ville, sont dans cette optique les conditions nécessaires à l'établissement d'un État de Droit. Or l'existence d'une milice - qui plus est décrite comme composée de criminels de guerre - contrôlant une partie de la ville, reste pour eux la preuve que cet État de droit n'existe pas au nord, du fait de l'obstruction des dirigeants serbes, mais également de l'inefficacité des Nations unies, nous y reviendrons. Elle leur est d'autant plus insupportable que l'UÇK a déposé les armes et s'est partiellement recyclée en corps de sécurité civile (le TMK), réévaluant son propre objectif de constitution d'une armée nationale.

Ensuite, la demande d'extension de la Zone de Confiance fait partie d'une revendication d'un autre ordre, celle d'avoir accès aux soins dispensés à l'hôpital de Mitrovica/Mitrovicë, une fois que cet hôpital sera redevenu multiethnique. Là encore, l'interprétation que l'on peut faire de ces revendications est double. Le caractère résolument " démocratique " des revendications (liberté de circulation, libre accès aux soins médicaux et non-discrimination quant à leur délivrance, établissement d'un État de Droit dans la zone nord, retour des personnes déplacées et réfugiées dans leurs anciennes demeures) donne effectivement toute légitimité aux attentes des Albanais qui les formulent. Elle est en adéquation avec la philosophie générale de la " bonne gouvernance " que les institutions internationales désirent voir appliquer, de même qu'elles se basent sur la lettre de la résolution 1244 du Conseil de Sécurité des Nations unies. Pour autant, elles pourraient aussi servir à légitimer des visées nettement moins avouables, que désigne la représentation de l'encerclement qui est active chez les Serbes de Mitrovica/Mitrovicë.

Se basant sur les événements passés - de "l'éradication " de la présence non-albanaise au sud de la ville au harcèlement régulier auquel sont soumis les Serbes des enclaves ou parfois ceux de Mitrovica/Mitrovicë nord - on pourrait interpréter ces volontés d'établir l'autorité de la communauté internationale au nord comme faisant partie d'une stratégie d'élimination de la présence serbe au nord de l'Ibar ${ }^{46}$. En effet, le harcèlement des communautés minoritaires constituerait un moyen relativement efficace de faire fuir les habitants " indésirables ", tout en laissant faisant apparaître ces actions comme des actes isolés, liés à des éléments extrémistes incontrôlables, et donc continuer à se ménager des soutiens internationaux. 
Enfin, il n'est pas non plus exclu que ce type d'action répréhensible soit effectivement le fait d'une minorité extrémiste active, plaçant les élites politiques albanaises dans une situation délicate, malgré la volonté de " bien faire ".

On le voit, les " bonnes volontés " et " bonnes paroles " peuvent éventuellement faire l'objet d'un double discours, à tout le moins d'effets pervers aboutissant à la réalisation de projets géopolitiques que semblent vouloir éviter les diverses composantes de la communauté internationale. Sur un autre plan, les revendications albanaises sur le nord de la ville, justement parce qu'elle sont légitimes sur le plan de la résolution 1244, génèrent une forte dévalorisation de l'activité de la communauté internationale. Les tentatives répétées, et toutes mises en échec, de la part de la MINUK de s'établir au nord de la ville, y compris par la force, aboutissent à ce qu'elle soit considérée comme incapable de gérer cette question. Les questions répétées et insistantes des journalistes albanais à la suite des incidents d'avril 2002 à Mitrovica/Mitrovicë révélaient cette frustration. " À quand le retour de la communauté internationale à Mitrovicë nord ? " était en substance une interrogation sous forme d'ultimatum posé à la crédibilité des Nations unies, dans la mesure où toutes les organisations internationales se refusaient à se rendre dans le nord de la ville suite à ces incidents, pour des raisons de sécurité. Conséquence logique de cette frustration politique forte, la municipalité demanda un accroissement de son autorité sur les forces de police. Un autre point faisait l'objet d'une attention particulière, l'immobilisme de la KFOR lors de ces mêmes événements et la position relativement ambiguë des autorités militaires sur le sujet de la coopération avec les élites autoproclamées de Mitrovica/Mitrovicë nord. Le fait que les soldats n'aient pas bougé alors même que les forces de la MINUK police étaient

prises sous le feu des miliciens serbes est apparu à bien des Albanais, mais aussi à des membres de la communauté internationale, comme un signe de leur " préférence " accordée aux Serbes. Si cette représentation de la KFOR ne semble pas généralisée parmi la population, la question des répercussions de cette position reste posée.

\section{LA PRÉSENCE INTERNATIONALE EN QUESTION}

Les différences que nous avons pu décrire entre les représentations de la KFOR et celles de la MINUK, exprimées par les acteurs locaux, ne sont pas imaginaires. L'arbitrage entre le militaire et le politique dans la gestion internationale de la ville de Mitrovica/Mitrovicë est ainsi révélateur de l'importance et de l'autonomie des acteurs militaires dans ce contexte particulier de postcrise. L'autonomie des acteurs militaires est double à Mitrovica/Mitrovicë. En effet, les aires de responsabilité qui découpent le Kosovo en cinq zones 
militaires 47 obéissent toutes au quartier général de la KFOR situé à Priština/Prishtinë dépendant directement de l'état-major de l'OTAN. Pour autant, l'attribution de ces aires de responsabilité à des Nations-Cadres, si elle permet une plus grande cohérence et efficacité de l'action militaire par le dispositif de contingent pilote $4^{8}$, laisse l'état-major multinational sans pouvoir réel, et surtout sans capacité de mettre fin aux différences stratégiques entre les nations contributrices, de sorte que l'on aboutisse à un commandement multinational à minima 49 . Le caractère national de la conduite des opérations et stratégies militaires au sein d'une force multinationale est ainsi un premier facteur d'autonomie forte des armées à Mitrovica/Mitrovicë. Ensuite, la faiblesse des rapports et des liens institutionnels entre les composantes civiles (MINUK mais aussi OSCE) et militaires de la présence internationale, notamment au niveau des commandements de brigade, aboutit à ce que les acteurs agissent sur un même espace sans coordination, laissant leurs stratégies diverger et parfois même se concurrencer.

Ce fut particulièrement le cas à Mitrovica/Mitrovicë entre la MINUK et la KFOR française. De fait, la MINUK a eu pour but depuis son arrivée de prendre pied au nord de la ville, là où le contrôle politique lui échappait. La stratégie des Nations unies s'est traduite par une alternance de coups de force et de déclarations rassurantes pour les Serbes, avec pour ambition principale de devenir auprès des populations un interlocuteur plus légitime que les élites autoproclamées. L'extension de la Zone de Confiance a ainsi été conclue sans négociation avec les Serbes au début de l'année 2000, avant d'être reportée devant l'hostilité des autorités militaires françaises. De même, la tentative de capture d'un des gardiens du pont en avril 2002 obéissait à cette logique du coup de force destiné à mettre un terme au contrôle des milices. Dans le même temps, une annexe de la municipalité de la MINUK venait d'ouvrir dans la partie serbe de la ville, offrant une alternative au leadership local en tendant la main aux populations civiles. Le plan en sept points du représentant spécial du secrétaire général Steiner pour Mitrovica/Mitrovicë présenté le $1^{\mathrm{er}}$ octobre 2002 montrait également un visage plus compréhensif de la communauté internationale, par rapport à l'intransigeance de la volonté de libre-circulation, et reprenait un certain nombre d'éléments satisfaisant partiellement des revendications serbes, comme le point quatre : la décentralisa-

47 Le nord du Kosovo est confié à la France, le centre-est (dont Priština/Prishtinë) à la Grande Bretagne, le sud-est aux États-Unis, le sud aux Allemands, et l'ouest aux Italiens.

$4^{8}$ Général de Courtivron, "Les interventions sous mandat international : le cas du Kosovo ", Relations Internationales et Stratégiques, (49), printemps 2003.

49 Voir Thomann (Jean-Claude), "Le Kosovo ", Défense Nationale, (10), octobre 2000 ; ainsi que Braem (Yann), Les relations Armées - ONG, des relations de pouvoir ? Caractéristiques et enjeux de la coopération civilo-militaire française: le cas du Kosovo (op.cit.), 2004. 
tion municipale (le fait que certaines décisions puissent être prises au niveau sub-municipal, en adaptant les décisions du conseil municipal à des intérêts d'un autre ordre de grandeur, celui des quartiers et des villages); mais plus encore le premier point : le principe de non-incursion (" To people living in the North, I give my solemn guarantie that there will be no incursion from the South (...). UNMIK Police and KFOR shall watch the bridge ")50. Néanmoins, et en dépit de ces garanties, la situation lors des années suivantes ne semble guère avoir changé.

Du point de vue de la KFOR, la stratégie était différente. Si, dans un premier temps, il semble que la volonté des autorités militaires ait été d'agir en vue d'empêcher l'installation des milices serbes ${ }^{51}$, suite à la militarisation de la rivière Ibar et de l'hôpital dès son arrivée à Mitrovica/Mitrovicë, il semble qu'elle se soit très rapidement cantonnée à son rôle de contrôle sécuritaire de la ville. Le refus d'étendre la Zone de Confiance peut, de ce point de vue, être interprété comme un refus d'accroître une zone de contrôle militaire extrêmement dense, nécessitant des ressources supplémentaires en hommes et matériel, alors que l'optique prioritaire reste le retrait le plus tôt possible. Elle peut, par ailleurs, s'expliquer dans la mesure où elle aurait été l'occasion d'un accroissement des tensions entre les communautés. Enfin, on ne peut négliger un facteur décisif : l'appropriation par les militaires des représentations géopolitiques serbes de l'encerclement ${ }^{52}$. Plusieurs entretiens ont ainsi montré que la KFOR envisageait cette représentation comme un scénario crédible, et que le précaire équilibre des forces que l'extension de la Zone de Confiance remettait en cause était susceptible de donner un avantage aux extrémistes albanais. Finalement, les autorités militaires ont multiplié les contacts avec l'élite politique de Mitrovica/Mitrovicë, notamment par le biais des médecins militaires se rendant régulièrement à l'hôpital, se ménageant alors des appuis en vue de garder la situation relativement calme au nord de la ville. Dans l'op-

\footnotetext{
$5^{\circ}$ Steiner (Michael), "A choice for Mitrovica : the seven point plan ", UNMIK, 01/10/2002.
}

${ }^{51}$ L'Opération Ibar, engagée le 20 février 2000 suite à une série d'incidents meurtriers impliquant des membres des deux communautés, avait pour but de désarmer la population serbe et les milices. Des contingents d'intervention américains, français, allemands, italiens et britanniques se sont rendus au nord de la ville pour y pratiquer des fouilles. Le bataillon américain a rencontré une très forte hostilité et a dû rebrousser chemin sans pouvoir effectuer sa mission (ICG, « Kosovo's Linchpin : Overcoming division in Mitrovica n (art.cit.) ; interviews à Mitrovica, 2001 et 2002).

52 Outre le fait que cette représentation géopolitique peut effectivement correspondre à une stratégie pensée par certains Albanais, on ne peut ignorer le fait que dans le Kosovo d'après-guerre, le rôle des " victimes " est tenu par les minorités non-albanaises. Leur situation d'enclavement, la pauvreté des espaces de peuplement minoritaire, et la brutalité avec laquelle certaines de ses minorités ont été expulsées de chez elles n'ont pu que contribuer à la victimisation des Serbes. Sans parler de " proserbisme " traditionnel des armées françaises, là encore une représentation géopolitique simpliste qui ne rend pas compte de la réalité, les entretiens que nous avons pu obtenir sur ces questions on traduit une sensibilité particulière des soldats à la détresse dans laquelle se trouvent les populations serbes, alors que les Albanais recevaient toutes les attentions de la communauté internationale. 
tique militaire, donc, il semble que l'objectif de sécurisation a joué en défaveur du règlement politique de la crise, que la MINUK s'évertuait en vain à mettre en œuvre.

L'absence de convergence stratégique, entre la stratégie politique de légitimation des autorités civiles de la MINUK, et la stratégie de sécurisation du territoire par la KFOR, est liée à la faiblesse des liens institutionnels entre ces différents acteurs. Outre le fait qu'en vertu de la Résolution 1244, la MINUK n'ait pas autorité sur les forces de l'OTAN, il convient ici de rappeler que les organisations internationales constituent pour les forces armées un environnement, tout comme la population civile du reste. Cette notion d'environnement est différente d'une notion de " partenariat " 53 , plus encore de hiérarchie, qui nécessiterait l'établissement de liens organiques. Plus que l'absence de convergences stratégiques, il apparait que ce sont parfois de véritables oppositions qui peuvent être constatées. L'épisode d'avril 2002 est encore ici révélateur de certaines tensions entre la MINUK et la KFOR à Mitrovica/Mitrovicë. À la suite de l'échec de l'opération de police menée par la MINUK sur le côté nord de l'Ibar, cette dernière a décrété une restriction de mouvement sur l'ensemble du nord de Mitrovica/Mitrovicë pour les personnels des organisations internationales, mais aussi ceux des ONG d'aide humanitaires 54 . Plus que d'une mesure de sécurité55, il s'agissait ici pour les Nations unies de signifier aux Serbes leur mécontentement et d'introduire une forme de conditionnalité à l'aide huma-

53 Pour une présentation des théories des relations civilo-militaires en contexte de post-conflit, voir Ankersen (Christopher), Coordination, cooperation, or something else: A framework for assessing power relations in the discourse of civil-military cooperation in peace support, Kingston : Inter University Seminar on Armed Forces and Society, Octobre 2002. Sur la question plus spécifique des relations civilo-militaires au Kosovo et de l'émergence de la coopération civilo-militaire en France, voir Braem (Yann), "French military and NGOs : recent trends in CIMIC construction ", Communication lors de la Conférence Biennale de l'Inter-University Seminar on Armed Forces and Society, Chicago 24-26 octobre 2003; ainsi que Braem (Yann), Les relations Armées - ONG, des relations de pouvoir ? Caractéristiques et enjeux de la coopération civilo-militaire française: le cas du Kosovo (op.cit.). De fait, malgré la prise en compte accrue d'une fonction civile des armées, incarnées par le rôle du cadre de contact (voir Maillet (Léandre), " Le rôle du cadre de contact ", La métamorphose des missions ? Le soldats et les armées dans le nouveaux contextes d'intervention, Paris : C2SD, 2000) et de l'action civilo-militaire, il nous apparait que l'environnement reste pour les armées un actant plus qu'un ensemble d'acteurs sur lesquels elles jouent en vue d'établir une stratégie de sortie de crise, et donc de désengagement. Ainsi l'aspect éloigné de " l'état final recherché n, équivalent français du Final End State américain, étroitement lié au plan politico-militaire, ne contribue pas à établir les liens essentiels entre activité militaire et activité politique, entre la gestion de la sécurité au quotidien et des objectifs politiques de plus long terme. À bien des points de vue, la conception traditionnelle des actions de guerre comme " continuation de la politique par d'autre moyens " reste la dimension majoritaire du cadre d'action militaire, alors que le rôle des armées dans les opérations de paix est celui du maintien de l'ordre, ce maintien devant contribuer au succès des stratégies politiques.

54 C'est alors l'UNHCR, bailleur de fond et agence opérationnelle des Nations unies, qui a décidé de prévenir les ONG que ses assurances ne couvriraient pas les dégâts matériels si toutefois une ONG se trouvait prise à partie au nord de l'Ibar.

55 Nous nous trouvions alors précisement au nord de la ville avec une ONG en infraction. 
nitaire apportée. Or la position de la KFOR au cours de ces événements différait largement de celle de la MINUK. Les hommes de la BMN nord considéraient effectivement l'opération de police comme une provocation contre les Serbes, qui avaient répondu de manière violente comme c'était, d'après eux, prévisible. Le fait que la BMN nord n'ai pas été prévenue de cette opération, signe d'un manque de confiance certain entre les deux acteurs, confirmait d'ailleurs pour bien des militaires français l'inefficacité caractérisée de la MINUK ${ }^{56}$ et sa volonté de leur nuire 57 . Or, face aux déclarations de la MINUK restreignant les mouvements au nord, les autorités militaires contredirent cette restriction, et firent largement connaître auprès des ONG d'aide humanitaire avec lesquelles elles étaient en contact que, d'après elles, le nord n'était pas dangereux.

Au-delà de l'anecdote, il nous apparaît que l'absence de cohérence entre les différentes composantes de la communauté internationale a pris à Mitrovica/Mitrovicë des proportions fortement contre-productives. D'une part, la communauté internationale est apparue divisée, au grand jour, voire parfois opposée, ce qui a contribué à décrédibiliser son action auprès des populations albanaises, mais aussi des populations serbes auprès desquelles la MINUK voulait apparaître comme une solution alternative crédible aux milices. D'autre part, elle a ménagé une marge de manœuvre non négligeable à des acteurs politiques dont la MINUK voulait justement se débarrasser, dans la mesure où ces acteurs ont continué à rester des interlocuteurs essentiels de la KFOR dans sa mission de sécurisation du territoire, notamment l'élite politique de l'hôpital. Enfin, cette absence de cohérence nous apparaît liée également à une absence de stratégie politique claire et définie sur plusieurs termes (courts, moyens et longs), permettant de gérer le statu-quo dans l'optique d'une vision politique.

\section{MITROVICA/MITROVICË, OU L'ILLUSTRATION DES DIFFICULTÉS DU NOUVEL INTERVENTIONNISME}

Nous avons pu le remarquer tout au long de ces pages, les questions de l'intervention, de la place de la communauté internationale et de sa philosophie de l'action sont posées plus ou moins directement, mais d'une manière profonde.

\footnotetext{
${ }^{56}$ La représentation de la MINUK comme une administration lourde, peuplée de bureaucrates incapables de s'adapter aux réalités du terrain est courante dans les cercles militaires comme humanitaires (Braem (Yann), art.cit.). Cette représentation s'appuie sur la différence ressentie entre l'homme de terrain, que sont les militaires en opération et les personnels humanitaires, et le fonctionnaire international, " gratte papier n, inefficace et présent au Kosovo pour des motivations financières.
}

57 Cette opération de police fut menée alors même qu'Alain Richard, ministre français de la Défense, se trouvait à Mitrovica/Mitrovicë. 
Mitrovica/Mitrovicë semble de ce point de vue concentrer des difficultés de plusieurs ordres ; pour un " nouvel interventionnisme humanitaire ${ }^{58}$ dont ce conflit fut le premier théâtre d'application, pour des questions plus générales, relatives aux interventions internationales sur les théâtres de guerre, mais aussi dans la mesure où cette ville est dans la situation géopolitique spécifique que nous avons décrite.

Le premier type de questions qui est posé de manière directe à Mitrovica/Mitrovicë, et que nous avons déjà en partie évoquées, reste celle des dynamiques territoriales propres à cet espace. La constitution d'une représentation frontalière, c'est-à-dire l'idée selon laquelle l'Ibar pourrait potentiellement dessiner le segment d'une future frontière entre la République de Serbie et un Kosovo indépendant, est le facteur majeur de tensions dans cette ville. Les représentations antagonistes traduisent de ce fait des projets géopolitiques posant la question de la validité des modes d'intervention et de gestion des espaces sous mandat international. C'est en quelque sorte le pari de la balkanisation qui fut joué au Kosovo. La balkanisation désignait, au début du vingtième siècle et suite aux guerres balkaniques, l'éclatement d'ensembles étatiques multinationaux en États nations, sous l'impulsion des grandes puissances 59 , la conflictualité étant d'autant plus importante que les revendications territoriales des peuples sont enchevêtrées ${ }^{60}$. L'intervention militaire de l'OTAN, de même que nombre d'éléments constituant les pierres angulaires de l'action de la MINUK sur place (processus électifs spécifiques à la Province, mise en place d'une juridiction spécifique au Kosovo, Cadre Constitutionnel de mai 2001) sont autant de signes de l'indépendance future du Kosovo.

Or, la stratégie la communauté internationale en cette matière reste officiellement le statu quo, en dépit des avancées significatives réalisées vers l'indépendance ${ }^{61}$. Cette question du statu quo amène plusieurs remarques. En effet, si l'horizon reste l'indépendance de la Province, la question reste posée des modalités de cette indépendance, et donc de la définition de la souverai-

${ }^{58}$ Debie (Franck), “ La communauté internationale et les Balkans n, in Yerasimos (Stéphane), éd., op.cit.

59 Pour une présentation cartographique des modifications de frontières liées à l'effondrement de l'Empire ottoman, voir Hupchick (Dennis P.), Cox (Harold E.), eds., A concise Historical Atlas of Eastern Europe, New York : St Martin Press, 1996; pour une présentation géopolitique, voir Yerasimos (Stéphane), Questions d'Orient, Paris : La Découverte, 1993. Pour une définition de la balkanisation, voir Yerasimos (Stéphane), "Le retour de la balkanisation dans son propre pays ", in Yerasimos (Stéphane), éd., op.cit. ; Lacoste (Yves), " Balkanisation ", Dictionnaire de Géopolitique (op.cit.) ; Krulic (Joseph), " La violence et la régulation de la violence dans l'espace yougoslave : réflexions critiques sur l'archéologie de la balkanisation ", communication in Études balkaniques : état des savoirs et pistes de recherche, Paris : Association Française d'Etude sur les Balkans, 19-20 décembre 2002.

${ }^{60}$ Lacoste (Yves), " Editorial ", Hérodote, (63), 4 e trimestre 1991.

${ }^{61}$ Rupnik (Jacques), art.cit.

${ }^{62}$ La théorie de la souveraineté limitée entend traduire une évolution historique, entre un État " westphalien " traditionnel, dont la souveraineté correspond à celle du XIX $\mathrm{e}^{\mathbf{e}}$ siècle, et un État " post-westpha- 
neté par les acteurs internationaux. S'il apparaît que la souveraineté limitée ${ }^{62}$, post-westphalienne ${ }^{63}$ semble bien être le cadre dans lequel la communauté internationale envisage la future indépendance du Kosovo, rien ne semble indiquer que les acteurs politiques locaux l'entendent sous le même sens ${ }^{64}$. Le pari de la balkanisation serait ainsi celui d'une réduction des dissonances cognitives autour de la notion de souveraineté. De ce point de vue pourtant, les événements ne semblent pas véritablement aller dans le sens d'une homogénéisation des points de vue entre les acteurs locaux et les acteurs internationaux. Bloquée dans cette impasse, la communauté internationale continue de reporter la question du statut final de la Province, préférant éviter tout débat et bloquant toute discussion sur ce point.

Toutefois, empêcher le débat n'est pas éliminer les idées qui l'animeraient s'il survenait. Et de ce point de vue, les questions territoriales traduisent le glissement, à travers des conflits sur le terrain, de représentations qui ne sont ni discutées, ni ouvertement débattues. En effet, ce statu-quo imposé ne signifie pas que les projets géopolitiques aient été abandonnés, Mitrovica/Mitrovicë restant l'un des points de confrontation de ces projets antagonistes. Plus encore, il semble que le statu-quo actuel encourage la poursuite de ces objectifs par d'autres moyens, plus insidieux que la discussion. Les stratégies de harcèlement dont se rendent coupables les extrémistes serbes tant qu'albanais obéissent aux mêmes logiques géopolitiques d'appropriation violente d'espaces géographiques plaçant les populations et leur localisation au cœur même du conflit, comme enjeux géopolitiques, à l'instar des opérations militaires serbes visant à vider le territoire du Kosovo au printemps 1999. Ces stratégies, et donc indirectement le statuquo, aboutissent en outre à légitimer des acteurs politiques dont la communauté internationale aimerait se passer, tels les Bridge-Watchers, "remparts serbes " contre "l'invasion albanaise ". De ce point de vue, il apparait assez nettement que le renforcement de la position d'un Marko Jakšić est étroitement lié à la longueur de la période sur laquelle il pu jouer la carte de la défense du territoire serbe et ainsi prétendre à des positions électives,

lien ", dont le mode d'exercice de la souveraineté, celui du XXI ${ }^{e}$ siècle, est lié à un ensemble de contraintes extérieures, les alliances régionales (le voisinage dans la terminologie de Keohane), le droit international, les ensembles supranationaux. Dans le cas qui nous intéresse, la souveraineté du XXI ${ }^{e}$ siècle impliquerait pour le Kosovo le respect des règles du Droit International Humanitaire et notamment de ses minorités non-albanaises, ainsi qu'un ensemble de contraintes de gouvernance. La souveraineté est dite post-westphalienne dans la mesure oủ la souveraineté de l'État, qui était la pierre angulaire du système international depuis le Traité de Westphalie, ne constitue plus désormais un obstacle à une intervention internationale légitime, notamment dans la mesure où elle vise à faire respecter les Droits de l'Homme.

${ }^{63}$ Rupnilk (Jacques), art.cit.; Keohane (Robert O.), " Political Authority after intervention : gradations in sovereignty ", in Keohane (Robert O.), Holzgrefe (J. L.), eds., op. cit.

64 Krulic (Joseph), art.cit. 
de la même manière que l'accroissement des scores du PDK dans la municipalité de Mitrovica/Mitrovicë nous semble corrélée à la frustration des électeurs albanais face à la perception de l'immobilisme de la communauté internationale.

De la même manière, la question du but à atteindre se pose lorsque l'on examine les stratégies d'acteurs impliqués dans cette géopolitique urbaine. Le lien entre le politique et le militaire se pose donc entre une gestion nécessaire de la sécurité au quotidien et une volonté politique n'apparaissant pas clairement. Dans ce cadre, les relations de pouvoir régissant l'interaction civilo-militaire laisse la part belle aux " fauteurs de troubles ", " seigneurs de la guerre " et autres " bad guys ". La question des modalités d'intervention dans une zone de post-conflit est de ce point de vue loin d'être réglée, au Kosovo comme dans d'autres parties du monde.

Finalement, si l'on rejoint les débats théoriques actuels sur la légitimité de l'interventionnisme, tout porte à croire que les réponses apportées aujourd'hui à cette question de la légitimité ne sont que partielles. Mitrovica/Mitrovicë, eu égard au nombre d'incidents interethniques violents qui l'agitent près de cinq années après l'intervention de l'OTAN, pose la question de l'efficacité des interventions internationales " humanitaires ". La légitimité de ces dernières, liées au fait qu'elle sauvent des vies ${ }^{65}$, ne répond que très partiellement aux défis de l'après-guerre. La question des interactions avec les logiques politiques locales reste donc de ce point de vue une des pierres angulaires des réflexions à mener lors des interventions militaires et autres opérations de paix de la communauté internationale.

65 Voir les définitions et démonstrations sur la légitimation de l'interventionnisme humanitaire apportées par Holzgrefe (J. L.), " The humanitarian debate ", in Keohane (Robert O.), Holzgrefe (J. L.), eds., op.cit. 